\title{
A High-Order Iterative Scheme for a Nonlinear Pseudoparabolic Equation and Numerical Results
}

\author{
Nguyen Huu Nhan $\mathbb{D}^{1,2,3}$ Tran Trinh Manh Dung, ${ }^{1,2}$ Le Thi Mai Thanh,, ${ }^{1,2,3}$ \\ Le Thi Phuong $\operatorname{Ngoc} \mathbb{D}^{1,2,4}$ and Nguyen Thanh Long $\mathbb{D}^{1,2}$ \\ ${ }^{1}$ Department of Mathematics and Computer Science, University of Science, 227 Nguyen Van Cu Street, District 5, \\ Ho Chi Minh City, Vietnam \\ ${ }^{2}$ Vietnam National University, Ho Chi Minh City, Vietnam \\ ${ }^{3}$ Nguyen Tat Thanh University, 300A Nguyen Tat Thanh Street, District 4, Ho Chi Minh City, Vietnam \\ ${ }^{4}$ University of Khanh Hoa, 01 Nguyen Chanh Street, Nha Trang, Vietnam \\ Correspondence should be addressed to Nguyen Thanh Long; longnt2@gmail.com
}

Received 22 September 2020; Revised 21 February 2021; Accepted 24 February 2021; Published 20 March 2021

Academic Editor: Bekir Sahin

Copyright @ 2021 Nguyen Huu Nhan et al. This is an open access article distributed under the Creative Commons Attribution License, which permits unrestricted use, distribution, and reproduction in any medium, provided the original work is properly cited.

\begin{abstract}
In this paper, by applying the Faedo-Galerkin approximation method and using basic concepts of nonlinear analysis, we study the initial-boundary value problem for a nonlinear pseudoparabolic equation with Robin-Dirichlet conditions. It consists of two main parts. Part 1 is devoted to proof of the unique existence of a weak solution by establishing an approximate sequence $\left\{u^{(m)}\right\}$ based on a $N$-order iterative scheme in case of $f \in C^{N}\left([0,1] \times\left[0, T^{*}\right] \times \mathbb{R}\right)(N \geq 2)$, or a single-iterative scheme in case of $f \in C^{1}\left(\bar{\Omega} \times\left[0, T^{*}\right] \times \mathbb{R}\right)$. In Part 2, we begin with the construction of a difference scheme to approximate $u^{(m)}$ of the $N$-order iterative scheme, with $N=2$. Next, we present numerical results in detail to show that the convergence rate of the 2-order iterative scheme is faster than that of the single-iterative scheme.
\end{abstract}

\section{Introduction}

In this paper, we consider the following initial-boundary problem:

$$
\begin{gathered}
u_{t}-\left(\mu(t)+\alpha(t) \frac{\partial}{\partial t}\right)\left(u_{x x}+\frac{1}{x} u_{x}\right)+\int_{0}^{t} g(t-s) \\
\cdot\left(u_{x x}(s)+\frac{1}{x} u_{x}(s)\right) \mathrm{d} s=f(x, t, u), \quad 1<x<R, 0<t<T, \\
u_{x}(1, t)-\zeta u(1, t)=u(R, t)=0, \\
u(x, 0)=\widetilde{u}_{0}(x),
\end{gathered}
$$

where $R>1$ and $\zeta \geq 0$ are given constants and $\mu(t), \alpha(t), f$, $g$, and $\widetilde{u}_{0}$ are given functions satisfying conditions specified later, with $u=u(x, t)$ being the unknown function.

Equation (1) is a form of the Sobolev-type differential equations; it is also called pseudoparabolic equation after Showalter's works [1-4] in the seventies. Since then, numerous interesting results for linear/nonlinear pseudoparabolic equations have been obtained. It is also well known that the Sobolev-type differential equations or the pseudoparabolic equations appear in the study of various problems of hydrodynamics, thermodynamics, and filtration theory, see [5-8] and the references therein. In the absence of the memory term in (1), i.e., $g=0$, the nonlinear pseudoparabolic problem of the types (1)-(3) is arisen in the investigations about second-grade or third-grade fluid flows, see 
$[9,10,11]$ and references therein. In [9], a mathematical model describing the unsteady flow of second-grade fluid in a circular cylinder is considered as follows:

$$
\begin{cases}\frac{\partial w}{\partial t}=\left(\nu+\alpha \frac{\partial}{\partial t}\right)\left(w_{r r}+\frac{1}{r} w_{r}\right)-N w, & 0<r<a, t>0, \\ w(a, t)=W, & t>0, \\ w(r, 0)=0, & 0 \leq r<a,\end{cases}
$$

where $w(r, t)$ is the velocity along the $z$-axis, $v$ is the kinematic viscosity, $\alpha$ is the material parameter, and $N$ is the imposed magnetic field. In the boundary and initial conditions, $W$ is the constant velocity at $r=a$ and $a$ is the radius of the cylinder. In the presence of the memory term in (1), i.e., $g \neq 0$, the problems of the types (1)-(3) are also studied in the theory of viscoelasticity, see [12]. Besides, it is well known that pseudoparabolic equations with nonlocal boundary conditions/nonlocal terms have been studied and many interesting results have been obtained such as stability, global existence, and finite time blow-up, for example, we refer to [13-19] and the references therein. In [15], Dai and Huang studied the solvability and the well-posedness of solutions for the nonlinear pseudoparabolic equation

$u_{t}+\left(a(x, t) u_{x t}\right)_{x}=F\left(x, t, u, u_{x}, u_{x x}\right), \quad \alpha<x<\beta, 0<t<T$, with the nonlocal moment boundary conditions $\int_{\alpha}^{\beta} u(x, t) \mathrm{d} x=\int_{\alpha}^{\beta} x u(x, t) \mathrm{d} x=0,0 \leq t \leq T$.

In [17], Sun et al. considered the Dirichlet problem for the nonlinear pseudoparabolic equation with a memory term as follows:

$$
\begin{cases}u_{t}-\Delta u-\Delta u_{t}+\int_{0}^{t} g(t-\tau) \Delta u(\tau) \mathrm{d} \tau=|u|^{p-2} u, & \text { in } \Omega \times(0, T), \\ u=0, & \text { on } \partial \Omega \times(0, T), \\ u(0)=u_{0}, & \text { in } \Omega,\end{cases}
$$

where $\Omega$ is a bounded domain of $\mathbb{R}^{n}(n \geq 1)$ with smooth boundary $\partial \Omega, \quad p>2, \quad T \in(0, \infty], \quad u_{0} \in H^{1}(\Omega) \quad$ and $g: \mathbb{R}_{+} \longrightarrow \mathbb{R}_{+}$is a positive nonincreasing function. The authors used the concavity method and the improved potential well method to obtain the global existence and the finite time blow-up phenomena of solutions.

This paper consists of two main parts. In Part 1, by using the $N$-order iterative method, Faedo-Galerkin method, and compact method, we prove existence and uniqueness of a weak solution of problems (1)-(3) (see Theorem 2). We begin with the establishment of the $N$-order nonlinear approximate sequence $\left\{u^{(m)}\right\}$ in case of $f \in C^{N}\left([0,1] \times\left[0, T^{*}\right] \times \mathbb{R}\right)$ via the $N$-order iterative scheme associated with problems (1)-(3) as follows:

$$
\left\{\begin{array}{l}
u^{(0)} \equiv 0, \\
u_{t}^{(m)}-\left(\mu(t)+\alpha(t) \frac{\partial}{\partial t}\right)\left(u_{x x}^{(m)}+\frac{1}{x} u_{x}^{(m)}\right)-\int_{0}^{t} g(t-s)\left(u_{x x}^{(m)}(s)+\frac{1}{x} u_{x}^{(m)}(s)\right) \mathrm{d} s \\
=\sum_{i=0}^{N-1} \frac{1}{i !} D_{3}^{i} f\left(x, t, u^{(m-1)}(x, t)\right)\left(u^{(m)}(x, t)-u^{(m-1)}(x, t)\right)^{i}, \\
u_{x}^{(m)}(1, t)-\zeta u^{(m)}(1, t)=u^{(m)}(R, t)=0, \\
u^{(m)}(0)=\widetilde{u}_{0},
\end{array}\right.
$$

and next, we prove that $\left\{u^{(m)}\right\}$ converges to the unique solution $u$ of problems (1)-(3) at a rate of order $N(N \geq 2)$; it means that $\left\|u^{(m)}-u\right\|_{X} \leq C\left\|u^{(m-1)}-u\right\|_{X}^{N}$, for some $C>0$, where $X$ is a suitable space. Scheme (7) is called the high- order iterative scheme or the $\mathrm{N}$-order iterative scheme. Specially, when $N=2$, the 2-order iterative scheme is given as follows:

$$
\left\{\begin{array}{l}
u^{(0)} \equiv 0 \\
u_{t}^{(m)}(t)-\mu(t) L u^{(m)}(t)-\alpha(t) L u_{t}^{(m)}(t)+\int_{0}^{t} g(t-s) L u^{(m)}(s) \mathrm{d} s \\
=f\left(x, t, u^{(m-1)}(x, t)\right)+D_{3} f\left(x, t, u^{(m-1)}(x, t)\right)\left(u^{(m)}(x, t)-u^{(m-1)}(x, t)\right) \\
u_{x}^{(m)}(1, t)-\zeta u^{(m)}(1, t)=u^{(m)}(R, t)=0 \\
u^{(m)}(0)=\widetilde{u}_{0}
\end{array}\right.
$$


where $L u=u_{x x}+(1 / x) u_{x}$. In case of $f \in C^{1}(\bar{\Omega} \times$ $\left.\left[0, T^{*}\right] \times \mathbb{R}\right)$, it is clear to see that the local existence and uniqueness of problems (1)-(3) also can be established by using the linear approximate sequence $\left\{u^{(m)}\right\}$ via a singleiterative scheme (see Remark 1). We note more that the abovementioned high-order iterative scheme is also used to obtain the existence of solutions in the previous papers [20-23]. In [23], Truong et al. studied the initial-boundary problem for a nonlinear wave equation of Kirchhoff-Carrier type. Here, by Galerkin method and compactness method, the existence and the convergence at $\mathrm{N}$-order rate of a recurrent sequence associated with the proposed problem were proved. Furthermore, when $N=3$, the 3 -order iterative scheme was established and solved numerically.

In this paper, the numerical results are also given in Part 2. First, this part is devoted to the construction of the difference scheme to approximate $u^{(m)}$ in the 2-order iterative scheme (8). In order to do this, we shall use a simple finitedifference scheme which is a standard model given in [24]. We first use the uniform partition $x_{i}=i h, h=\left(1 / N_{0}\right)$, $i=0,1, \ldots, N_{0}$, and the forward difference formulas (see [24], pages 36 and 43) to approximate the $k^{\text {th }}$ derivatives, $k=1,2$, in spatial variable, as follows:

$$
\begin{aligned}
u_{x}^{(m)}\left(x_{i}, t\right) & \simeq \frac{u_{i}^{(m)}(t)-u_{i-1}^{(m)}(t)}{h}, \\
u_{x}^{(m)}\left(x_{0}, t\right) & \simeq \frac{u_{1}^{(m)}(t)-u_{0}^{(m)}(t)}{h}, \\
u_{x x}^{(m)}\left(x_{i}, t\right) & \simeq \frac{u_{i-1}^{(m)}(t)-2 u_{i}^{(m)}(t)+u_{i+1}^{(m)}(t)}{h^{2}}, \\
L u_{i}^{(m)}(t) & =u_{x x}^{(m)}\left(x_{i}, t\right)+\frac{1}{x_{i}} u_{x}^{(m)}\left(x_{i}, t\right) \\
& \equiv a_{i} u_{i-1}^{(m)}(t)+b_{i} u_{i}^{(m)}(t)+\gamma u_{i+1}^{(m)}(t), \\
a_{i} & =\left(\frac{1}{h^{2}}-\frac{1}{x_{i} h}\right), \\
b_{i} & =\left(\frac{-2}{h^{2}}+\frac{1}{x_{i} h}\right), \\
\gamma & =\frac{1}{h^{2}}, \quad 2 \leq i \leq N_{0} .
\end{aligned}
$$

This is also a technique used in [20, 25-29]. After replacing $(9)_{1,2,3}$ in problem (8), we obtain the first-order integro-differential equation with a vector-function variable in the form as follows:

$$
\bar{A}(t) \frac{\mathrm{d} \vec{u}^{(m)}}{\mathrm{d} t}(t)+B^{(m)}(t) \vec{u}^{(m)}(t)+\int_{0}^{t} g(t-s) C \overrightarrow{\mathcal{u}}^{(m)}(s) \mathrm{d} s=\vec{F}^{(m)}(t),
$$

where $\bar{A}(t), B^{(m)}(t)$ are functional matrices depending on a time variable $t$ and $C \in \mathfrak{M}_{N_{0}}\left(\mathfrak{M}_{N_{0}}\right.$ is the set of real $N_{0}$-size matrices). Next, we make discretizations in time variables $t_{j}=j \Delta t, \Delta t=(T / M)$, and $j=0,1, \ldots, M$ and approximate the integral $\int_{0}^{t} g(t-s) C \vec{u}^{(m)}(s) \mathrm{d} s$ by the trapezoidal formula (see [24], page 56), and we remark that this technique was also used in $[26,27,30]$. Then, we obtain the following algorithm to determine the finite-difference approximate solutions of $u^{(m)}$ given by the 2-order iterative scheme formula (71)

$$
\begin{aligned}
\bar{A}_{0} \vec{u}_{1}^{(m)}= & \left(\bar{A}_{0}-\Delta t B_{0}^{(m)}\right) \vec{u}_{0}^{(m)}+\Delta t \vec{F}^{(m)}\left(t_{0}\right), \\
\bar{A}_{1} \vec{u}_{2}^{(m)}= & \left(\bar{A}_{1}-\Delta t B_{1}^{(m)}-\frac{1}{2}(\Delta t)^{2} g_{0} C\right) \vec{u}_{1}^{(m)} \\
& -\frac{1}{2}(\Delta t)^{2} g_{1} C \vec{u}_{0}^{(m)}+\Delta t \vec{F}^{(m)}\left(t_{1}\right), \\
\bar{A}_{j} \vec{u}_{j+1}^{(m)}= & \left(\bar{A}_{j}-\Delta t B_{j}^{(m)}-\frac{1}{2}(\Delta t)^{2} g_{0} C\right) \vec{u}_{j}^{(m)}-\frac{1}{2}(\Delta t)^{2} g_{1} C \vec{u}_{0}^{(m)} \\
& -(\Delta t)^{2} \sum_{\nu=1}^{j-1} g_{j-\nu} C \vec{u}_{\nu}^{(m)}+\Delta t \vec{F}^{(m)}\left(t_{j}\right), \quad 2 \leq j \leq M-1,
\end{aligned}
$$

where $\bar{A}_{j}=\bar{A}\left(t_{j}\right)$ and $B_{j}^{(m)}=B^{(m)}\left(t_{j}\right)$. Similarly, we have constructed the algorithm to find the finite-difference approximate solutions of $u^{(m)}$ given by the single-order iterative scheme (formula (92)).

It is well known that the finite-difference method to solve nonlinear elliptic/parabolic/pseudoparabolic equations and the consistency, accuracy, efficiency, stability, convergence, and the other properties of difference schemes are mentioned in many works [25-27, 29, 31-45].

In [31], Amirali et al. considered the following initialboundary value problem for the pseudoparabolic equation with delay

$$
\left\{\begin{array}{l}
L u:=\frac{\partial u}{\partial t}-\frac{\partial}{\partial x}\left(a(x, t) \frac{\partial^{2} u}{\partial t \partial x}\right)-\frac{\partial}{\partial x}\left(b(x, t) \frac{\partial u}{\partial x}\right) \\
=f(x, t, u(x, t), u(x, t)-r), \quad(x, t) \in Q, \\
u(0, t)=u(l, t)=0, \quad t \in(0, T], \\
u(x, t)=\varphi(x, t), \quad(x, t) \in \bar{Q} \times[-r, 0],
\end{array}\right.
$$

where $Q=(0, l) \times(0, T], r$ represents the delay parameter, $a(x, t) \geq \alpha>0, \quad|b(x, t)| \leq b^{*}, \quad \varphi(x, t), \quad$ and $f(x, t, u(x, t), u(x, t-r))$ are given sufficiently smooth functions satisfying certain regularity conditions. Here, the finite-difference technique was applied to the numerical solution of problem (12). By the method of integral identities with use of the piecewise linear basis functions in space and interpolating quadrature rules with weight and remainder term in integral forms, two-level difference scheme was constructed for singular perturbation cases without delay. The finite-difference discretization was shown to be absolutely stable and convergent of order two in space and of order one in time. Based on the method of energy estimates, the error analysis for the approximate solution was 
presented. The error estimates were obtained in the discrete norm. Some numerical results confirming the expected behavior of the method were shown.

In [32], Beshtokov studied the following nonlocal boundary value problem for a third-order pseudoparabolic equation with variable coefficients

$$
\begin{cases}u_{t}=L u+f(x, t), & 0<x<l, 0<t \leq T, \\ L_{0} u(0, t)=0, & 0 \leq t \leq T, \\ u(l, t)=\beta \int_{0}^{l} x^{m} u(x, t) \mathrm{d} x-\mu(t), & 0 \leq t \leq T, \\ u(x, 0)=u_{0}(x), & 0 \leq x \leq l,\end{cases}
$$

where

$$
\begin{aligned}
L u= & \frac{1}{x^{m}}\left(x^{m} k(x, t) u_{x}\right)_{x}+\frac{1}{x^{m}}\left(\eta(x, t) u_{x}\right)_{x t} \\
& +r(x, t) u_{x}-q(x, t) u, \\
L_{0} u= & k u_{x}+\left(\eta u_{x}\right)_{t} .
\end{aligned}
$$

The existence and uniqueness of the solution of problems (13) and (14) were proved by the Riemann function method. For its solution, in the differential and finite-difference settings, the author derived a priori estimates that implied the stability of the solution with respect to the initial data and the right-hand side on a layer as well as the convergence of the solution of the difference problem to the solution of the differential problem.

In [26], Jachimavičienè and Sapagovas studied the following two-dimensional pseudoparabolic equation:

$$
u_{t}=\Delta u+\eta \Delta u_{t}+f(x, y, t), \quad 0<x, y<1,0<t<T,
$$

with nonlocal integral boundary conditions

$$
\left\{\begin{array}{l}
u(0, y, t)=\gamma_{1} \int_{0}^{1} u(x, y, t) \mathrm{d} x+\mu_{1}(y, t) \\
u(1, y, t)=\gamma_{2} \int_{0}^{1} u(x, y, t) \mathrm{d} x+\mu_{2}(y, t) \\
u(x, 0, t)=\mu_{3}(x, t) \\
u(x, 1, t)=\mu_{4}(x, t)
\end{array}\right.
$$

and initial condition

$$
u(x, y, 0)=\varphi(x, y)
$$

where $f, \varphi, \mu_{i}$, and $i=1,2,3,4$ are given functions and $\eta, \gamma_{1}$, and $\gamma_{2}>0$ are given constants. They decomposed problem (15) into two locally one-dimensional problems from layer $t=t_{n}$ to layer $t=t_{n+1}$ as follows:

$$
\left\{\begin{array}{l}
\frac{1}{2} \frac{\partial u}{\partial t}=\frac{\partial^{2} u}{\partial x^{2}}+\eta \frac{\partial}{\partial t}\left(\frac{\partial^{2} u}{\partial x^{2}}\right)+\frac{1}{2} f, \quad t_{n} \leq t \leq t_{n+(1 / 2)}, \\
\frac{1}{2} \frac{\partial u}{\partial t}=\frac{\partial^{2} u}{\partial y^{2}}+\eta \frac{\partial}{\partial t}\left(\frac{\partial^{2} u}{\partial y^{2}}\right)+\frac{1}{2} f, \quad t_{n+(1 / 2)} \leq t \leq t_{n+1},
\end{array}\right.
$$

and next, they changed equation (18) to the following onedimensional difference schemes:

$$
\left\{\begin{array}{l}
\frac{u_{i j}^{n+(1 / 2)}-u_{i j}^{n}}{\tau}=\Lambda_{1} u_{i j}^{n+(1 / 2)}+\eta \frac{\Lambda_{1} u_{i j}^{n+(1 / 2)}-\Lambda_{1} u_{i j}^{n}}{(\tau / 2)}+\frac{1}{2} f_{i j}^{n+(1 / 2)}, \\
\frac{u_{i j}^{n+1}-u_{i j}^{n+(1 / 2)}}{\tau}=\Lambda_{2} u_{i j}^{n+1}+\eta \frac{\Lambda_{2} u_{i j}^{n+1}-\Lambda_{2} u_{i j}^{n+1 / 2}}{(\tau / 2)}+\frac{1}{2} f_{i j}^{n+1},
\end{array}\right.
$$

where $\Lambda_{1} u_{i j}^{n}=\left(\left(u_{i-1 j}^{n}-2 u_{i j}^{n}+u_{i+1 j}^{n}\right) / h^{2}\right)$ and $\Lambda_{2} u_{i j}^{n+1}=$ $\left(\left(u_{i j-1}^{n+1}-2 u_{i j}^{n+1}+u_{i j+1}^{n+1}\right) / h^{2}\right)$. They proved the difference equation (19) approximating the differential equation (18) with the truncation error $O\left(h^{2}+\tau\right)$. Moreover, if $\gamma_{1}+\gamma_{2}<2$, then the difference schemes (19) are stable for all values of $h$ and $\tau$.

In [34], Brachet and Chehab considered the following nonlinear parabolic equation:

$$
u_{t}+F(u)=0 \text {, }
$$

where $F: \mathbb{R}^{n} \longrightarrow \mathbb{R}^{n}$ is a regular map. The backward Euler scheme applied to the above equation generates the iterations

$$
u^{(k+1)}-u^{(k)}+\Delta t F\left(u^{(k+1)}\right)=0,
$$

and the nonlinear term $F\left(u^{(k+1)}\right)$ is approximated by

$$
F\left(u^{(k+1)}\right) \approx F\left(u^{(k)}\right)+F^{\prime}\left(u^{(k)}\right)\left(u^{(k+1)}-u^{(k)}\right) .
$$

Consequently, the following difference equation is established:

$$
u^{(k+1)}=u^{(k)}-\Delta t\left(I d+\Delta t F^{\prime}\left(u^{(k)}\right)\right)^{-1} F\left(u^{(k)}\right) .
$$

Stability results in the linear and the nonlinear case and numerical simulations of 2D incompressible Navier-Stokes equations for illustrating the robustness of the method were also presented here. It is clear that the approximation given by (22) is similar to the approximation of the nonlinear term on the left hand side of the 2-order iterative scheme (8).

In [41], the authors undeveloped two new B-spline collocation algorithms based on cubic trigonometric B-spline functions to find approximate solutions of a nonlinear parabolic partial differential equations with Dirichlet and Neumann boundary conditions. Some wellknown nonlinear parabolic problems were also solved here to check the applicability, accuracy, and efficiency of the proposed algorithms.

In [37], departing from a generalized Burgers-Huxley partial differential equation, the authors provided a Mickens-type, nonlinear, finite-difference discretization of the model. They proved that the method proposed also preserves many of the relevant characteristics of these solutions, such as the positivity, the boundedness, and the spatial and temporal monotonicity, and then, in [42], the authors established the property of convergence for a finite-difference discretization of a diffusive partial differential equation 
with generalized Burgers convective law and generalized Hodgkin-Huxley reaction. The authors proved that the method introduced in [37] was convergent with linear order in time and quadratic order in space. Some numerical experiments were provided in order to support the analytical results.

In this paper, at the end of Part 2, an illustrated example and the numerical results are detailed to show that the convergence rate of the 2-order iterative scheme is faster than that of the single-iterative scheme.

\section{Existence and Uniqueness}

Throughout this paper, we set $\Omega=(1, R)$ and use $L^{2}=$ $L^{2}(\Omega)$ to denote the Lebesgue space with the inner product defined by $(u, v)=\int_{1}^{R} u(x) v(x) \mathrm{d} x$, and $L^{2}$-norm of a function $u \in L^{2}$ is denoted by $\|u\|=\sqrt{(u, u)}$. We use $H^{m}=H^{m}(\Omega)$ to denote the Sobolev spaces with the norm $\|u\|_{H^{m}}=\left(\sum_{i=0}^{m}\left\|D^{i} u\right\|^{2}\right)^{(1 / 2)}$.

Moreover, we also introduce three weighted scalar products

$$
\begin{aligned}
\langle u, v\rangle & =\int_{1}^{R} x u(x) v(x) \mathrm{d} x, \quad u, v \in L^{2}, \\
\langle u, v\rangle_{1} & =\langle u, v\rangle+\left\langle u_{x}, v_{x}\right\rangle, \quad u, v \in H^{1}, \\
\langle u, v\rangle_{2} & =\langle u, v\rangle+\left\langle u_{x}, v_{x}\right\rangle+\left\langle u_{x x}, v_{x x}\right\rangle, \quad u, v \in H^{2},
\end{aligned}
$$

and then, $L^{2}, H^{1}$, and $H^{2}$ are the Hilbert spaces with respect to the abovementioned scalar products. We denote $\|u\|_{0}=\sqrt{\langle u, u\rangle}, \quad u \in L^{2} ; \quad\|u\|_{1}=\sqrt{\langle u, u\rangle}, \quad u \in H^{1} ;$ $\|u\|_{2}=\sqrt{\langle u, u\rangle_{2}}, u \in H^{2}$.

Put

$$
V=\left\{v \in H^{1}: v(R)=0\right\} .
$$

The symmetric bilinear form $a(\cdot, \cdot)$ is defined by

$$
a(u, w)=\left\langle u_{x}, w_{x}\right\rangle+\zeta u(1) w(1), \quad \text { for all } u, w \in V,
$$

with $\zeta \geq 0$ being a given constant and $\|v\|_{a}=\sqrt{a(v, v)}$. Then, we have the following lemmas.

Lemma 1. The imbeddings $V \longmapsto C^{0}(\bar{\Omega})$ are compact and

(i) $\|v\|_{C^{0}(\bar{\Omega})} \leq \sqrt{R-1}\left\|v_{x}\right\|_{0} \leq \sqrt{R-1}\|v\|_{a}$, for all $v \in V$,

(ii) $\|v\|_{0} \leq \frac{\sqrt{2 R}(R-1)}{2}\left\|v_{x}\right\|_{0}, \quad$ for all $v \in V$,

(iii) $\left\|v_{x}\right\|_{0} \leq\|v\|_{a} \leq \sqrt{1+\zeta(R-1)}\left\|v_{x}\right\|_{0}, \quad$ for all $v \in V$.
Lemma 2. The symmetric bilinear form $a(\cdot, \cdot)$ is continuous on $V \times V$ and coercive on $V$, i.e., there exist two positive constants $C_{0}$ and $C_{1}$ such that

$$
\begin{aligned}
& \text { (i) }|a(u, v)| \leq C_{1}\left\|u_{x}\right\|_{0}\left\|v_{x}\right\|_{0}, \\
& \text { (ii) } a(v, v) \geq C_{0}\left\|v_{x}\right\|_{0}^{2},
\end{aligned}
$$

for all $u, v \in V$. Moreover, $C_{1}=1+\zeta(R-1)$ and $C_{0}=1$.

The notation $\|\cdot\|_{X}$ is the norm in the Banach space $X$, and $X^{\prime}$ is the dual space of $X$. We denote by $L^{p}(0, T ; X)$, $1 \leq p \leq \infty$, for the Banach space of functions $u:(0, T) \longrightarrow X$ measurable, such that

$$
\begin{aligned}
& \|u\|_{L^{p}(0, T ; X)}=\left(\int_{0}^{T}\|u(t)\|_{X}^{p} \mathrm{~d} t\right)^{(1 / p)}<\infty, \text { for } 1 \leq \mathrm{p}<\infty, \\
& \|u\|_{L^{\infty}(0, T ; X)}=\underset{0<t<T}{\operatorname{esssup}}\|u(t)\|_{X}, \quad \text { for } \mathrm{p}=\infty .
\end{aligned}
$$

Denote $\quad u(t)(x)=u(x, t), \quad \dot{u}(t)=u^{\prime}(t)=u_{t}(t)$ $=(\partial u / \partial t)(t), u_{x}(t)=(\partial u / \partial x)(t)$, and $u_{x x}=\left(\partial^{2} u / \partial x^{2}\right)(t)$. With $f \in C^{N}\left([0,1] \times\left[0, T^{*}\right] \times \mathbb{R}\right), f=f(x, t, u)$, we put $D_{1} f=(\partial f / \partial x), \quad D_{2} f=(\partial f / \partial t), \quad D_{3} f=(\partial f / \partial u) \quad$ and $D^{\alpha} f=D_{1}^{\alpha_{1}} D_{2}^{\alpha_{2}} D_{3}^{\alpha_{3}} f, \quad \alpha=\left(\alpha_{1}, \alpha_{2}, \alpha_{3}\right) \in \mathbb{Z}_{+}^{3}, \quad|\alpha|=\alpha_{1}+\alpha_{2}+$ $\alpha_{3} \leq N, D^{0} f=D^{(0,0,0)} f=f$.

For a fixed constant $T^{*}>0$, we make the following assumptions:

$$
\begin{aligned}
\left(H_{1}\right) & \widetilde{u}_{0} \in V \cap H^{2}, \widetilde{u}_{0 x}(1)-\zeta \widetilde{u}_{0}(1)=0 ; \\
\left(H_{2}\right) & g \in L^{2}\left(0, T^{*}\right) ; \\
\left(H_{3}\right) & \alpha \in H^{1}\left(0, T^{*}\right), \alpha(t) \geq \alpha_{*}>0, \quad \text { for all } t \in\left[0, T^{*}\right] ; \\
\left(H_{4}\right) & \mu \in H^{1}\left(0, T^{*}\right), \mu(t) \geq \mu_{*}>0, \quad \text { for all } t \in\left[0, T^{*}\right] ; \\
\left(H_{5}\right) \quad & f \in C^{1}\left(\bar{\Omega} \times\left[0, T^{*}\right] \times \mathbb{R}\right) \text { satisfies the conditions } \\
& D_{3}^{i} f, D_{2} D_{3}^{j} f \in C^{0}\left(\bar{\Omega} \times\left[0, T^{*}\right] \times \mathbb{R}\right), \\
& 1 \leq i \leq N, 1 \leq j \leq N-1 .
\end{aligned}
$$

Definition 1. The weak solution of problems (1)-(3) is a function $u \in C\left([0, T] ; V \cap H^{2}\right) \quad$ such that $u^{\prime} \in L^{\infty}\left(0, T ; V \cap H^{2}\right)$ and $u$ satisfies the following variational equation:

$$
\left\{\begin{array}{l}
\left\langle u^{\prime}(t), w\right\rangle+\alpha(t) a\left(u^{\prime}(t), w\right)+\mu(t) a(u(t), w) \\
=\int_{0}^{t} g(t-s) a(u(s), w) \mathrm{d} s+\langle f[u](t), w\rangle, \quad \text { for all } w \in V, \text { a.e., } t \in(0, T), \\
u(0)=\tilde{u}_{0},
\end{array}\right.
$$


where $f[u](x, t)=f(x, t, u(x, t))$. For each $T \in\left(0, T^{*}\right]$, we define $\quad W_{T}=\left\{v \in C\left([0, T] ; V \cap H^{2}\right): v^{\prime} \in L^{\infty}(0, T ; V\right.$ $\left.\left.\cap H^{2}\right)\right\}$, and then $W_{T}$ is a Banach space with the norm $\|v\|_{W_{T}}=\max \left\{\|v\|_{C\left([0, T] ; V \cap H^{2}\right)},\left\|v^{\prime}\right\|_{L^{\infty}\left(0, T: V \cap H^{2}\right)}\right\}$. For $M>0$, we put $B_{T}(M)=\left\{v \in W_{T}:\|v\|_{W_{T}} \leq M\right\}$.

Now, we construct the recurrent sequence $\left\{u^{(m)}\right\}$ defined by $u^{(0)} \equiv 0$, and suppose that

$$
u^{(m-1)} \in B_{T}(M) .
$$

Then, $u^{(m)}$ is found by the fact that $u^{(m)} \in B_{T}(M), m \geq 1$, and $u^{(m)}$ satisfies

$$
\left\{\begin{array}{l}
\left\langle\dot{u}^{(m)}(t), w\right\rangle+\alpha(t) a\left(\dot{u}^{(m)}(t), w\right)+\mu(t) a\left(u^{(m)}(t), w\right) \\
=\int_{0}^{t} g(t-s) a\left(u^{(m)}(s), w\right) \mathrm{d} s+\left\langle\mathscr{F}^{(m)}(t), w\right\rangle, \quad \text { for all } w \in V, \text { a.e., } t \in(0, T), \\
u^{(m)}(0)=\widetilde{u}_{0},
\end{array}\right.
$$

where

$$
\mathscr{F}^{(m)}(x, t)=\sum_{i=0}^{N-1} \frac{1}{i !} D_{3}^{i} f\left(x, t, u^{(m-1)}(x, t)\right)\left(u^{(m)}(x, t)-u^{(m-1)}(x, t)\right)^{i} .
$$

Using the standard Faedo-Galerkin method, which is introduced by Lions in [44], we can prove the following theorem.

Theorem 1. Assume that $\widetilde{u}_{0}, g, \alpha(t), \mu(t)$, and $f$ satisfy the conditions $\left(H_{1}\right)-\left(H_{5}\right)$, respectively, then there exist the constants $M>0$ and $T>0$ such that problems (33), (34) admit $u^{(m)} \in B_{T}(M)$.

By using Theorem 1 and the compact imbedding theorems, we shall prove the existence and uniqueness of weak local solution in time to problems (1)-(3).

First, we consider the space

$$
W_{1}(T)=\left\{v \in C([0, T] ; V): v^{\prime} \in L^{2}(0, T ; V)\right\},
$$

then $W_{1}(T)$ is a Banach space with respect to the norm (see [44]) $\|v\|_{W_{1}(T)}=\|v\|_{C([0, T] ; V)}+\left\|v^{\prime}\right\|_{L^{2}(0, T ; V)}$.
Theorem 2. Let $\left(H_{1}\right)-\left(H_{5}\right)$ hold. Then, there exist constants $M>0$ and $T>0$ such that problems (1)-(3) have a unique weak solution $u \in B_{T}(M)$ and the recurrent sequence $\left\{u^{(m)}\right\}$, defined by (32)-(34), converges at a rate of order $N$ to the solution $u$ strongly in the space $W_{1}(T)$ in the sense

$$
\left\|u^{(m)}-u\right\|_{W_{1}(T)} \leq C\left\|u^{(m-1)}-u\right\|_{W_{1}(T)}^{N},
$$

for all $m \geq 1$, where $C$ is a suitable constant. On the other hand, the following estimate is fulfilled:

$$
\left\|u^{(m)}-u\right\|_{W_{1}(T)} \leq C_{T}\left(k_{T}\right)^{N^{m}}, \quad \text { for all } m \in \mathbb{N},
$$

where $C_{T}$ and $0<k_{T}<1$ are the constants depending only on $T$.

Proof. We shall prove that $\left\{u^{(m)}\right\}$ is a Cauchy sequence in $W_{1}(T)$.

First, we put $v^{(m)}=u^{(m+1)}-u^{(m)}$. Then, $v^{(m)}$ satisfies the variational problem,

$$
\left\{\begin{array}{l}
\left\langle\dot{v}^{(m)}(t), w\right\rangle+\alpha(t) a\left(\dot{v}^{(m)}(t), w\right)+\mu(t) a\left(v^{(m)}(t), w\right) \\
=\int_{0}^{t} g(t-s) a\left(v^{(m)}(s), w\right) \mathrm{d} s+\left\langle\mathscr{F}^{(m+1)}(t)-\mathscr{F}^{(m)}(t), w\right\rangle, \quad \forall w \in V, \\
v^{(m)}(0)=0,
\end{array}\right.
$$

where $\mathscr{F}^{(m)}(x, t)$ is defined by (34).

Taking $w=\dot{v}^{(m)}(t)$ in (38), after integrating in $t$, we have

$$
\begin{aligned}
Z_{m}(t) & =2 \int_{0}^{t} \mu l(s)\left\|v^{(m)}(s)\right\|_{a}^{2} \mathrm{~d} s+2 \int_{0}^{t} \mathrm{~d} \tau \int_{0}^{\tau} g(\tau-s) a\left(v^{(m)}(s), \dot{v}^{(m)}(\tau)\right) \mathrm{d} s+2 \int_{0}^{t}\left\langle\mathscr{F}^{(m+1)}(s)-\mathscr{F}^{(m)}(s), \dot{v}^{(m)}(s)\right\rangle \mathrm{d} s \\
& =J_{1}+J_{2}+J_{3},
\end{aligned}
$$


where

$$
Z_{m}(t)=\mu(t)\left\|v^{(m)}(t)\right\|_{a}^{2}+2 \int_{0}^{t}\left(\left\|\dot{v}^{(m)}(s)\right\|_{0}^{2}+\alpha(s)\left\|\dot{v}^{(m)}(s)\right\|_{a}^{2}\right) \mathrm{d} s .
$$

Next, we have to estimate the integrals on the right-hand side of (40).

We put $\left.K_{M}(f)=\|f\|_{C^{0}(\bar{\Omega}} \quad{ }_{M}\right)+\sum_{i=1}^{N}\left\|D_{3}^{i} f\right\|_{C^{0}\left(\bar{\Omega}_{M}\right)}$ $+\sum_{j=1}^{N-1}\left\|D_{2} D_{3}^{j} f\right\|_{C^{0}\left(\bar{\Omega}_{M}\right)}$, where

$\|f\|_{C^{0}\left(\bar{\Omega}_{M}\right)}=\sup \left\{|f(x, t, y)|:(x, t, y) \in \bar{\Omega}_{M}\right\}$,

$$
\bar{\Omega}_{M}=[1, R] \times\left[0, T^{*}\right] \times[-\sqrt{R-1} M, \sqrt{R-1} M] .
$$

Using the inequality $Z_{m}(t) \geq \beta_{*}\left[\left\|v^{(m)}(t)\right\|_{a}^{2}\right.$ $\left.+2 \int_{0}^{t}\left(\left\|\dot{v}^{(m)}(s)\right\|_{0}^{2}+\left\|\dot{v}^{(m)}(s)\right\|_{a}^{2}\right) \mathrm{d} s\right]$, with $\beta_{*}=\min \left\{\mu_{*}, \alpha_{*}\right\}$, the integrals $J_{1}$ and $J_{2}$ are estimated as follows:

$$
\begin{aligned}
J_{1} & =2 \int_{0}^{t} \mu \prime(s)\left\|v^{(m)}(s)\right\|_{a}^{2} \mathrm{~d} s \leq \frac{1}{\beta_{*}}\|\mu \prime\|_{L^{\infty}\left(0, T^{*}\right)} \int_{0}^{t} Z_{m}(s) \mathrm{d} s \\
J_{2} & =2 \int_{0}^{t} \mathrm{~d} \tau \int_{0}^{t} g(\tau-s) a\left(v^{(m)}(s), \dot{v}^{(m)}(\tau)\right) \mathrm{d} s \\
& \leq \frac{1}{4} Z_{m}(t)+\frac{2 T^{*}\|g\|_{L^{2}\left(0, T^{*}\right)}^{2}}{\beta_{*}} \int_{0}^{t} Z_{m}(s) \mathrm{d} s
\end{aligned}
$$

Using Taylor's expansion of the functions $f\left(x, t, u^{(m)}\right)=$ $f\left(x, t, u^{(m-1)}+v^{(m-1)}\right)$ around the point $u^{(m-1)}$ up to order $N$, we obtain

$$
f\left(x, t, u^{(m)}\right)-f\left(x, t, u^{(m-1)}\right)=\sum_{i=1}^{N-1} \frac{1}{i !} D_{3}^{i} f\left(x, t, u^{(m-1)}\right)\left(v^{(m-1)}\right)^{i}+\frac{1}{N !} D_{3}^{N} f\left(x, t, \bar{u}^{(m)}\right)\left(v^{(m-1)}\right)^{N},
$$

where $\bar{u}^{(m)}=u^{(m-1)}(x, t)+\theta v^{(m-1)}(x, t), 0<\theta<1$.

Hence,

$$
\mathscr{F}^{(m+1)}(t)-\mathscr{F}^{(m)}(t)=\sum_{i=1}^{N-1} \frac{1}{i !} D_{3}^{i} f\left(x, t, u^{(m)}\right)\left(v^{(m)}\right)^{i}+\frac{1}{N !} D_{3}^{N} f\left(x, t, \bar{u}^{(m)}\right)\left(v^{(m-1)}\right)^{N}
$$

Note that

$$
\begin{gathered}
\left|v^{(m)}(x, t)\right|^{i} \leq\left(\sqrt{R-1}\left\|v^{(m)}(t)\right\|_{a}\right)^{i} \leq(\sqrt{R-1})^{i}(2 M)^{i-1} \sqrt{\frac{Z_{m}(t)}{\beta_{*}}}, \\
\left|v^{(m-1)}(x, t)\right|^{N} \leq\left(\sqrt{R-1}\left\|v^{(m-1)}(t)\right\|_{a}\right)^{N} \leq(\sqrt{R-1})^{N}\left\|v^{(m-1)}\right\|_{W_{1}(T)}^{N} .
\end{gathered}
$$

Therefore, we have

$$
\begin{aligned}
\left\|\mathscr{F}^{(m+1)}(t)-\mathscr{F}^{(m)}(t)\right\|_{0} \leq & \left(\frac{R^{2}-1}{2}\right) K_{M}(f) \sum_{i=1}^{N-1} \frac{1}{i !}(\sqrt{R-1})^{i}(2 M)^{i-1} \sqrt{\frac{Z_{m}(t)}{\beta_{*}}} \\
& +\frac{1}{N !}\left(\frac{R^{2}-1}{2}\right) K_{M}(f)(\sqrt{R-1})^{N}\left\|v^{(m-1)}\right\|_{W_{1}(T)}^{N} \\
\equiv & \bar{\gamma}_{1}(M) \sqrt{Z_{m}(t)}+\bar{\gamma}_{2}(M)\left\|v^{(m-1)}\right\|_{W_{1}(T)}^{N}
\end{aligned}
$$


where $\bar{\gamma}_{1}(M)=\left(\left(R^{2}-1\right) / 2\right) K_{M}(f) \sum_{i=1}^{N-1}(1 / i !)\left(\left((\sqrt{R-1})^{i}\right.\right.$ $\left.\left.(2 M)^{i-1}\right) / \sqrt{\beta_{*}}\right), \quad \bar{\gamma}_{2}(M)=(1 / N !)\left(\left(R^{2}-1\right) / 2\right) K_{M} \quad(f)$ $(\sqrt{R-1})^{N}$. Since the above inequality, the integral $J_{3}$ can be estimated by

$$
\begin{aligned}
J_{3} & =2 \int_{0}^{t}\left\langle\mathscr{F}^{(m+1)}(s)-\mathscr{F}^{(m)}(s), \dot{v}^{(m)}(s)\right\rangle \mathrm{d} s \\
& \leq 2 \int_{0}^{t}\left\|\mathscr{F}^{(m+1)}(s)-\mathscr{F}^{(m)}(s)\right\|_{0}^{2} \mathrm{~d} s+\frac{1}{2} \int_{0}^{t}\left\|\dot{v}^{(m)}(s)\right\|_{0}^{2} \mathrm{~d} s \\
& \leq 4 T \bar{\gamma}_{2}^{2}(M)\left\|v^{(m-1)}\right\|_{W_{1}(T)}^{2 N}+4 \bar{\gamma}_{1}^{2}(M) \int_{0}^{t} Z_{m}(s) \mathrm{d} s+\frac{1}{4} Z_{m}(t) .
\end{aligned}
$$

By (39) and (42), it follows from (47) that

$$
Z_{m}(t) \leq 8 T \bar{\gamma}_{2}^{2}(M)\left\|v^{(m-1)}\right\|_{W_{1}(T)}^{2 N}+2 \bar{\gamma}_{3}(M) \int_{0}^{t} Z_{m}(s) \mathrm{d} s,
$$

where $\quad \bar{\gamma}_{3}(M)=4 \bar{\gamma}_{1}^{2}(M)+\left(\left(\|\mu \prime\|_{L^{\infty}\left(0, T^{*}\right)}+2 T^{*}\|g\|_{L^{2}\left(0, T^{*}\right)}^{2}\right)\right.$ $\left./ \beta_{*}\right)$.

Using Gronwall's Lemma, we deduce from (48) that

$$
\left\|v^{(m)}\right\|_{W_{1}(T)} \leq \mu_{T}\left\|v^{(m-1)}\right\|_{W_{1}(T)}^{N}, \quad \forall m \in \mathbb{N},
$$

where $\mu_{T}=(1+(1 / \sqrt{2}))\left((2 \sqrt{2}) / \sqrt{\beta_{*}}\right) \bar{\gamma}_{2}(M) \sqrt{T} \exp \left(T \bar{\gamma}_{3}\right.$ $(M))$.

Choosing $T>0$ small enough such that $k_{T}=M\left(\mu_{T}\right)^{(1 /(N-1))}<1$, it follows from (49) that, for all $m$ and $p$,

$$
\left\|u^{(m)}-u^{(m+p)}\right\|_{W_{1}(T)} \leq \frac{1}{\left(\mu_{T}\right)^{(1 /(N-1))}\left(1-k_{T}\right)}\left(k_{T}\right)^{N^{m}} .
$$

The above inequality ensures that $\left\{u^{(m)}\right\}$ is a Cauchy sequence in $W_{1}(T)$. Then, there exists $u \in W_{1}(T)$ such that

$$
u^{(m)} \longrightarrow u \text { strongly in } W_{1}(T) .
$$

Note that $u^{(m)} \in B_{T}(M)$, then there exists a subsequence $\left\{u^{\left(m_{j}\right)}\right\}$ of $\left\{u^{(m)}\right\}$ such that

$$
\left\{\begin{array}{l}
u^{\left(m_{j}\right)} \longrightarrow u, \quad \text { in } L^{\infty}\left(0, T ; V \cap H^{2}\right) \text { weakly }{ }^{*}, \\
\dot{u}^{\left(m_{j}\right)} \longrightarrow u^{\prime}, \quad \text { in } L^{\infty}\left(0, T ; V \cap H^{2}\right) \text { weakly }{ }^{*}, \\
u \in B_{T}(M) .
\end{array}\right.
$$

We note that

$$
\begin{aligned}
& \left|\mathscr{F}^{(m)}(x, t)-f\left[u^{(m-1)}\right](x, t)\right| \leq K_{M}(f) \sum_{i=1}^{N-1} \frac{1}{i !}\left(\sqrt{R-1}\left\|u^{(m)}-u^{(m-1)}\right\|_{W_{1}(T)}\right)^{i}, \\
& \left|f\left[u^{(m-1)}\right](x, t)-f[u](x, t)\right| \leq K_{M}(f) \sqrt{R-1}\left\|u^{(m-1)}-u\right\|_{W_{1}(T)} .
\end{aligned}
$$

Hence,

$$
\begin{aligned}
\left\|\mathscr{F}^{(m)}-f[u]\right\|_{C^{0}\left(\overline{Q_{T}}\right)} & \leq\left\|\mathscr{F}^{(m)}-f\left[u^{(m-1)}\right]\right\|_{C^{0}\left(\overline{Q_{T}}\right)}+\left\|f\left[u^{(m-1)}\right]-f[u]\right\|_{C^{0}\left(\overline{Q_{T}}\right)} \\
& \leq K_{M}(f) \sum_{i=1}^{N-1} \frac{1}{i !}(\sqrt{R-1})^{i}\left\|u^{(m)}-u^{(m-1)}\right\|_{W_{1}(T)}^{i}+K_{M}(f) \sqrt{R-1}\left\|u^{(m-1)}-u\right\|_{W_{1}(T)} .
\end{aligned}
$$

Therefore, we deduce from (51) and (54) that

$$
\mathscr{F}^{(m)} \longrightarrow f[u] \text { strongly in } C^{0}\left(\overline{Q_{T}}\right) .
$$

Letting $m=m_{j} \longrightarrow \infty$ in (33) and (34) and using (51, 52 , and 55), it implies that there exists $u \in B_{T}(M)$ satisfying (31). The proof of the existence is completed. Next, it is not difficult to prove the uniqueness of a solution of (31). Afterward, by passing to the limit in (50) as $p \longrightarrow \infty$ for fixed $m$, we get (37). Theorem 2 is proved completely.

Remark 1. The local existence and uniqueness of problems (1)-(3) can be established by using the linear approximate sequence $\left\{u^{(m)}\right\}$ corresponding to schemes (32)-(34) with $\mathscr{F}^{(m)}(x, t)=f\left(x, t, u^{(m-1)}(x, t)\right)$. Then, the assumption for $f$ is weakened as follows: $f \in C^{1}\left(\bar{\Omega} \times\left[0, T^{*}\right] \times \mathbb{R}\right)$.

We note more that the scheme obtained here is called the single-iterative scheme.

\section{Numerical Scheme}

In this section, we first construct a difference scheme to approximate the solution $u$ of problems (1)-(3) via approximating $u^{(m)}$ in the 2 -order iterative scheme (8). It is implied from Theorems 1 and 2 that, $u^{(m)}$ is definite by 
problems (33) and (34) with the nonlinear term $\mathscr{F}^{(m)}(x, t)$ given on right-hand side of (33) as follows:

$\mathscr{F}^{(m)}(x, t)=f\left(x, t, u^{(m-1)}(x, t)\right)+b^{(m)}(x, t)\left(u^{(m)}(x, t)-u^{(m-1)}(x, t)\right)$,

with

$$
b^{(m)}(x, t)=D_{3} f\left(x, t, u^{(m-1)}(x, t)\right) .
$$

\section{Putting}

$$
u_{i}^{(m)}(t)=u^{(m)}\left(x_{i}, t\right), \quad x_{i}=1+i h, i=0,1, \ldots, N+1, h=\frac{R-1}{N+1} .
$$

Rewriting (8) at the node $x=x_{i}$,

$$
\begin{cases}\dot{u}_{i}^{(m)}(t)-\alpha(t) L \dot{u}_{i}^{(m)}(t)-\mu(t) L u_{i}^{(m)}(t)+\int_{0}^{t} g(t-s) L u_{i}^{(m)}(s) \mathrm{d} s-b_{i}^{(m)}(t) u_{i}^{(m)}(t)=F_{i}^{(m)}(t), & 0 \leq i \leq N, 0<t<T \\ u_{x}^{(m)}\left(x_{0}, t\right)-\zeta u_{0}^{(m)}(t)=u_{N+1}^{(m)}(t)=0, & 0 \leq i \leq N+1, \\ u_{i}^{(m)}(0)=u^{(m)}\left(x_{i}, 0\right)=\widetilde{u}_{0}\left(x_{i}\right)=\widetilde{u}_{0 i}, & \end{cases}
$$

in which

$$
\begin{aligned}
& b_{i}^{(m)}(t)=b^{(m)}\left(x_{i}, t\right)=D_{3} f\left(x_{i}, t, u_{i}^{(m-1)}(t)\right), \\
& F_{i}^{(m)}(t)=F^{(m)}\left(x_{i}, t\right)=f\left(x_{i}, t, u_{i}^{(m-1)}(t)\right)-b_{i}^{(m)}(t) u_{i}^{(m-1)}(t) .
\end{aligned}
$$

Replacing the derivatives in spatial variable $x$ of (59) by the following approximations (see [24], pages 36 and 43),

$$
\begin{aligned}
u_{x}^{(m)}\left(x_{i}, t\right) & \simeq \frac{u_{i}^{(m)}(t)-u_{i-1}^{(m)}(t)}{h}, \\
u_{x}^{(m)}\left(x_{0}, t\right) & \simeq \frac{u_{1}^{(m)}(t)-u_{0}^{(m)}(t)}{h}, \\
u_{x x}^{(m)}\left(x_{i}, t\right) & \simeq \frac{u_{i-1}^{(m)}(t)-2 u_{i}^{(m)}(t)+u_{i+1}^{(m)}(t)}{h^{2}}, \\
L u_{i}^{(m)}(t) & =u_{x x}^{(m)}\left(x_{i}, t\right)+\frac{1}{x_{i}} u_{x}^{(m)}\left(x_{i}, t\right) \\
& \simeq \frac{u_{i-1}^{(m)}(t)-2 u_{i}^{(m)}(t)+u_{i+1}^{(m)}(t)}{h^{2}}+\frac{1}{x_{i}} \frac{u_{i}^{(m)}(t)-u_{i-1}^{(m)}(t)}{h} \\
& =\left(\frac{1}{h^{2}}-\frac{1}{x_{i} h}\right) u_{i-1}^{(m)}(t)+\left(\frac{-2}{h^{2}}+\frac{1}{x_{i} h}\right) u_{i}^{(m)}(t)+\frac{1}{h^{2}} u_{i+1}^{(m)}(t) \\
& \equiv a_{i} u_{i-1}^{(m)}(t)+b_{i} u_{i}^{(m)}(t)+\gamma u_{i+1}^{(m)}(t),
\end{aligned}
$$

with

$$
\begin{aligned}
a_{i} & =\left(\frac{1}{h^{2}}-\frac{1}{x_{i} h}\right), \\
b_{i} & =\left(\frac{-2}{h^{2}}+\frac{1}{x_{i} h}\right), \\
\gamma & =\frac{1}{h^{2}}, \quad 2 \leq i \leq N .
\end{aligned}
$$

$$
\begin{cases}\dot{u}_{i}^{(m)}(t)-\alpha(t)\left[a_{i} \dot{u}_{i-1}^{(m)}(t)+b_{i} \dot{u}_{i}^{(m)}(t)+\gamma \dot{u}_{i+1}^{(m)}(t)\right]-\mu(t)\left[a_{i} u_{i-1}^{(m)}(t)+b_{i} u_{i}^{(m)}(t)+\gamma u_{i+1}^{(m)}(t)\right] \\ +\int_{0}^{t} g(t-s)\left[a_{i} u_{i-1}^{(m)}(s)+b_{i} u_{i}^{(m)}(s)+\gamma u_{i+1}^{(m)}(s)\right] \mathrm{d} s-b_{i}^{(m)}(t) u_{i}^{(m)}(t)=F_{i}^{(m)}(t), \quad 1 \leq i \leq N, \\ \frac{u_{1}(t)-u_{0}(t)}{h}-\zeta u_{0}(t)=u_{N+1}(t)=0 & 0 \leq i \leq N . \\ u_{i}(0)=u\left(x_{i}, 0\right)=\widetilde{u}_{0}\left(x_{i}\right)=\widetilde{u}_{0 i}, & \end{cases}
$$


Using the boundary conditions (63) $)_{2}$ with $u_{0}^{(m)}(t)=\left(\left(u_{1}^{(m)}(t)\right) /(1+h \zeta)\right), u_{N+1}(t)=0$ and after eliminating the unknown functions $u_{0}(t)$ and $u_{N+1}(t)$ in the first align $i=1$ and the last align $i=N$, respectively, then system (63) is rewritten as follows:

$$
\left\{\begin{array}{l}
\dot{u}_{1}^{(m)}(t)-\alpha(t)\left[\widetilde{a}_{1} \dot{u}_{1}^{(m)}(t)+\gamma \dot{u}_{2}^{(m)}(t)\right]-\mu(t)\left[\widetilde{a}_{1} u_{1}^{(m)}(t)+\gamma u_{2}^{(m)}(t)\right] \\
+\int_{0}^{t} g(t-s)\left[\widetilde{a}_{1} u_{1}^{(m)}(s)+\gamma u_{2}^{(m)}(s)\right] \mathrm{d} s-b_{1}^{(m)}(t) u_{1}^{(m)}(t)=F_{1}^{(m)}(t), \quad i=1, \\
\dot{u}_{i}^{(m)}(t)-\alpha(t)\left[a_{i} \dot{u}_{i-1}^{(m)}(t)+b_{i} \dot{u}_{i}^{(m)}(t)+\gamma \dot{u}_{i+1}^{(m)}(t)\right]-\mu(t)\left[a_{i} u_{i-1}^{(m)}(t)+b_{i} u_{i}^{(m)}(t)+\gamma u_{i+1}^{(m)}(t)\right] \\
+\int_{0}^{t} g(t-s)\left[a_{i} u_{i-1}^{(m)}(s)+b_{i} u_{i}^{(m)}(s)+\gamma u_{i+1}^{(m)}(s)\right] \mathrm{d} s \\
-b_{i}^{(m)}(t) u_{i}^{(m)}(t)=F_{i}^{(m)}(t), \quad 2 \leq i \leq N-1, \\
\dot{u}_{N}^{(m)}(t)-\alpha(t)\left[a_{N} \dot{u}_{N-1}^{(m)}(t)+b_{N} \dot{u}_{N}^{(m)}(t)\right]-\mu(t)\left[a_{N} u_{N-1}^{(m)}(t)+b u_{N}^{(m)}(t)\right] \\
+\int_{0}^{t} g(t-s)\left[a_{N} u_{N-1}^{(m)}(s)+b u_{N}^{(m)}(s)\right] \mathrm{d} s-b_{N}^{(m)}(t) u_{N}^{(m)}(t)=F_{N}^{(m)}(t), \quad i=N,
\end{array}\right.
$$

where $\tilde{a}_{1}=\left(a_{1} /(1+h \zeta)\right)+b_{1}$.

Using (64) to rewrite (63) into a vector align as follows:

$$
\left\{\begin{array}{l}
\bar{A}(t) \frac{\mathrm{d} \vec{u}^{(m)}}{\mathrm{d} t}(t)+B^{(m)}(t) \vec{u}^{(m)}(t)+\int_{0}^{t} g(t-s) C \vec{u}^{(m)}(s) \mathrm{d} s=\vec{F}^{(m)}(t), \\
\vec{u}^{(m)}(0)=\left(u_{1}^{(m)}(0), \ldots, u_{N}^{(m)}(0)\right)^{T}=\left(\widetilde{u}_{0}\left(x_{1}\right), \ldots, \widetilde{u}_{0}\left(x_{N}\right)\right)^{T},
\end{array}\right.
$$

where

$$
\left\{\begin{array}{l}
\vec{u}^{(m)}(t)=\left(u_{1}^{(m)}(t), \ldots, u_{N}^{(m)}(t)\right)^{T}=\left(u^{(m)}\left(x_{1}, t\right), \ldots, u^{(m)}\left(x_{N}, t\right)\right)^{T}, \\
\vec{F}^{(m)}(t)=\left(F_{1}^{(m)}(t), \ldots, F_{N}^{(m)}(t)\right)^{T},
\end{array}\right.
$$
and $\bar{A}(t), B^{(m)}(t), C \in \mathfrak{M}_{N}\left(\mathfrak{M}_{N}\right.$ is the set of real $N$-order matrices) are defined by

$$
\begin{aligned}
& C=\left[\begin{array}{ccccccc}
\tilde{a}_{1} & \gamma & & & & & \\
a_{2} & b_{2} & \gamma & & & & \\
& a_{3} & b_{3} & \gamma & & & \\
& & \ddots & \ddots & \ddots & & \\
& & & \ddots & \ddots & \ddots & \\
& & & & a_{N-1} & b_{N-1} & \gamma \\
& & & & & a_{N} & b_{N}
\end{array}\right], \\
& \widetilde{B}_{m}(t)=\left[\begin{array}{lllll}
b_{1}^{(m)}(t) & & & & \\
& b_{2}^{(m)}(t) & & & \\
& & \ddots & & \\
& & & \ddots & \\
& & & & b_{N}^{(m)}(t)
\end{array}\right] \\
& \bar{A}(t)=I-\alpha(t) C, \\
& B^{(m)}(t)=-\widetilde{B}_{m}(t)-\mu(t) C .
\end{aligned}
$$

Approximating the derivatives $\left(\left(\mathrm{d} \vec{u}^{(m)}\right) / \mathrm{d} t\right)\left(t_{j}\right)$ by the differences in time variable and the following partition, 


$$
\begin{aligned}
\frac{\mathrm{d} \vec{u}^{(m)}}{\mathrm{d} t}\left(t_{j}\right) & \simeq \frac{\vec{u}_{j+1}^{(m)}-\vec{u}_{j}^{(m)}}{\Delta t}, \\
\vec{u}_{j}^{(m)} & =\vec{u}^{(m)}\left(t_{j}\right), \quad t_{j}=j \Delta t, j=0, \ldots, M, \Delta t=\frac{T}{M}, \\
\bar{A}_{j} & =\bar{A}\left(t_{j}\right), \\
B_{j}^{(m)} & =B^{(m)}\left(t_{j}\right),
\end{aligned}
$$

then the align (65) was rewritten as follows:

$$
\bar{A}_{j} \frac{\vec{u}_{j+1}^{(m)}-\vec{u}_{j}^{(m)}}{\Delta t}+B_{j}^{(m)} \vec{u}_{j}^{(m)}+\int_{0}^{t_{j}} g\left(t_{j}-s\right) C \vec{u}^{(m)}(s) \mathrm{d} s=\vec{F}^{(m)}\left(t_{j}\right) .
$$

Note that, the integral $\int_{0}^{t_{j}} g\left(t_{j}-s\right) C \vec{u}^{(m)}(s) \mathrm{d} s$ can be approximated by the trapezoidal formula (see [24], page 56) as follows:

$$
\int_{0}^{t_{j}} g\left(t_{j}-s\right) C \vec{u}^{(m)}(s) \mathrm{d} s \simeq \begin{cases}\Delta t\left(\frac{g_{1} C \vec{u}_{0}^{(m)}+g_{0} C \vec{u}_{1}^{(m)}}{2}\right), & j=1, \\ \Delta t\left(\frac{g_{j} C \vec{u}_{0}^{(m)}+g_{0} C \vec{u}_{j}^{(m)}}{2}+\sum_{\nu=1}^{j-1} g_{j-\nu} C \vec{u}_{\nu}^{(m)}\right), & 2 \leq j \leq M,\end{cases}
$$

where $g_{j}=g\left(t_{j}\right), 0 \leq j \leq M$.

Hence, align (69) can be rewritten as follows:

$$
\begin{aligned}
\bar{A}_{0} \vec{u}_{1}^{(m)} & =\left(\bar{A}_{0}-\Delta t B_{0}^{(m)}\right) \vec{u}_{0}^{(m)}+\Delta t \vec{F}^{(m)}\left(t_{0}\right) \equiv \bar{b}_{1}^{(m)}, \\
\bar{A}_{1} \vec{u}_{2}^{(m)} & =\left(\bar{A}_{1}-\Delta t B_{1}^{(m)}-\frac{1}{2}(\Delta t)^{2} g_{0} C\right) \vec{u}_{1}^{(m)}-\frac{1}{2}(\Delta t)^{2} g_{1} C \vec{u}_{0}^{(m)}+\Delta t \vec{F}^{(m)}\left(t_{1}\right) \equiv \bar{b}_{2}^{(m)}, \\
\bar{A}_{j} \vec{u}_{j+1}^{(m)} & =\left(\bar{A}_{j}-\Delta t B_{j}^{(m)}-\frac{1}{2}(\Delta t)^{2} g_{0} C\right) \vec{u}_{j}^{(m)}-\frac{1}{2}(\Delta t)^{2} g_{1} C \vec{u}_{0}^{(m)}-(\Delta t)^{2} \sum_{\nu=1}^{j-1} g_{j-\nu} C \vec{u}_{\nu}^{(m)}+\Delta t \vec{F}^{(m)}\left(t_{j}\right) \\
& \equiv \bar{b}_{j+1}^{(m)}, \quad 2 \leq j \leq M-1,
\end{aligned}
$$

in which

$$
\left\{\begin{array}{l}
\vec{u}^{(m)}(t)=\left(u_{1}^{(m)}(t), \ldots, u_{N}^{(m)}(t)\right)^{T}=\left(u^{(m)}\left(x_{1}, t\right), \ldots, u^{(m)}\left(x_{N}, t\right)\right)^{T}, \\
\vec{u}_{j}^{(m)}=\vec{u}^{(m)}\left(t_{j}\right)=\left(u^{(m)}\left(x_{1}, t_{j}\right), \ldots, u^{(m)}\left(x_{N}, t_{j}\right)\right)^{T}, \\
\vec{u}_{0}^{(m)}=\vec{u}^{(m)}(0)=\left(\widetilde{u}_{0}\left(x_{1}\right), \ldots, \widetilde{u}_{0}\left(x_{N}\right)\right)^{T}, \\
\vec{F}^{(m)}\left(t_{j}\right)=\left(F_{1}^{(m)}\left(t_{j}\right), \ldots, F_{N}^{(m)}\left(t_{j}\right)\right)^{T}, \\
F_{i}^{(m)}\left(t_{j}\right)=F^{(m)}\left(x_{i}, t_{j}\right)=f\left(x_{i}, t_{j}, u_{i}^{(m-1)}\left(t_{j}\right)\right)-b_{i}^{(m)}\left(t_{j}\right) u_{i}^{(m-1)}\left(t_{j}\right), \\
b_{i}^{(m)}\left(t_{j}\right)=b^{(m)}\left(x_{i}, t_{j}\right)=\frac{\partial f}{\partial u}\left(x_{i}, t_{j}, u_{i}^{(m-1)}\left(t_{j}\right)\right) .
\end{array}\right.
$$

Description of scheme (71):
(A) Let $M, N$ to be fixed constants. At the first iteration with $m=0$, we set up the given vector

$$
\begin{aligned}
\vec{u}_{j}^{(0)} & =\vec{u}^{(0)}\left(t_{j}\right) \\
& =\left(u_{1}^{(0)}\left(t_{j}\right), \ldots, u_{N}^{(0)}\left(t_{j}\right)\right)^{T} \\
& =\left(u^{(0)}\left(x_{1}, t_{j}\right), \ldots, u^{(0)}\left(x_{N}, t_{j}\right)\right)^{T} \equiv 0, \quad j=1, \ldots, M .
\end{aligned}
$$

(B) At the $(m-1)^{\text {th }}$ iteration, suppose that we have

$$
\begin{aligned}
\vec{u}_{j}^{(m-1)} & =\vec{u}^{(m-1)}\left(t_{j}\right) \\
& =\left(u_{1}^{(m-1)}\left(t_{j}\right), \ldots, u_{N}^{(m-1)}\left(t_{j}\right)\right)^{T}, \quad j=1, \ldots, M .
\end{aligned}
$$


(C) Then, the vectors $\vec{u}_{j}^{(m)}=\vec{u}^{(m)}\left(t_{j}\right)=\left(u_{1}^{(m)}\right.$ $\left.\left(t_{j}\right), \ldots, u_{N}^{(m)}\left(t_{j}\right)\right)^{T}, j=1, \ldots, M$ can be computed consecutively by the following steps.

(C1) The computation of $\vec{u}_{1}^{(m)}=\left(u_{1}^{(m)}\left(t_{1}\right), \ldots\right.$, $\left.u_{N}^{(m)}\left(t_{1}\right)\right)^{T}$.

(i) With the first given vector $\vec{u}_{0}^{(m)}=\left(\widetilde{u}_{0}\left(x_{1}\right), \ldots, \widetilde{u}_{0}\left(x_{N}\right)\right)^{T}$, we calculate the matrices

$$
\begin{aligned}
\bar{A}_{0} & =\bar{A}\left(t_{0}\right)=I-\alpha\left(t_{0}\right) C, \\
B^{(m)}\left(t_{0}\right) & =-\widetilde{B}_{m}\left(t_{0}\right)-\mu\left(t_{0}\right) C,
\end{aligned}
$$

and the vectors

$$
\begin{aligned}
\vec{F}^{(m)}\left(t_{0}\right) & =\left(F_{1}^{(m)}\left(t_{0}\right), \ldots, F_{N}^{(m)}\left(t_{0}\right)\right)^{T}, \\
\bar{b}_{1}^{(m)} & =\left(\bar{A}_{0}-\Delta t B_{0}^{(m)}\right) \vec{u}_{0}^{(m)}+\Delta t \vec{F}^{(m)}\left(t_{0}\right) .
\end{aligned}
$$

(ii) Finding the vector $\vec{u}_{1}^{(m)}=\left(u_{1}^{(m)}\left(t_{1}\right), \ldots, u_{N}^{(m)}\left(t_{1}\right)\right)^{T}$ by solving the following system

$$
\bar{A}_{0} \vec{u}_{1}^{(m)}=\bar{b}_{1}^{(m)}
$$

(C2) The computation of $\vec{u}_{2}^{(m)}=\left(u_{1}^{(m)}\left(t_{2}\right), \ldots, u_{N}^{(m)}\right.$ $\left.\left(t_{2}\right)\right)^{T}$.

(i) Calculating the matrices

$$
\begin{aligned}
\bar{A}_{1} & =\bar{A}\left(t_{1}\right)=I-\alpha\left(t_{1}\right) C, \\
B^{(m)}\left(t_{1}\right) & =-\widetilde{B}_{m}\left(t_{1}\right)-\mu\left(t_{1}\right) C,
\end{aligned}
$$

and the vectors

$$
\begin{aligned}
\vec{F}^{(m)}\left(t_{1}\right)= & \left(F_{1}^{(m)}\left(t_{1}\right), \ldots, F_{N}^{(m)}\left(t_{1}\right)\right)^{T} \\
\bar{b}_{2}^{(m)}= & \left(\bar{A}_{1}-\Delta t B_{1}^{(m)}-\frac{1}{2}(\Delta t)^{2} g_{0} C\right) \vec{u}_{1}^{(m)} \\
& -\frac{1}{2}(\Delta t)^{2} g_{1} C \vec{u}_{0}^{(m)}+\Delta t \vec{F}^{(m)}\left(t_{1}\right) .
\end{aligned}
$$

(ii) Finding the vector $\vec{u}_{2}^{(m)}=\left(u_{1}^{(m)}\left(t_{2}\right), \ldots, u_{N}^{(m)}\left(t_{2}\right)\right)^{T}$ by solving the following system

$$
\bar{A}_{1} \vec{u}_{2}^{(m)}=\bar{b}_{2}^{(m)}
$$

(C3) The computation of $\vec{u}_{j+1}^{(m)}=\left(u_{1}^{(m)}\left(t_{j+1}\right), \ldots, u_{N}^{(m)}\right.$ $\left.\left(t_{j+1}\right)\right)^{T}$. Suppose that the vectors $\vec{u}_{1}^{(m)}, \vec{u}_{2}^{(m)}, \ldots$, $\vec{u}_{j}^{(m)}$ are calculated; we determine the vector $\vec{u}_{j+1}^{(m)}=\left(u_{1}^{(m)}\left(t_{j+1}\right), \ldots, u_{N}^{(m)}\left(t_{j+1}\right)\right)^{T}$ by recurrence as follows:

(i) Calculating the matrices

$$
\begin{array}{r}
\bar{A}_{j}=\bar{A}\left(t_{j}\right)=I-\alpha\left(t_{j}\right) C, \\
B^{(m)}\left(t_{j}\right)=-\widetilde{B}_{m}\left(t_{j}\right)-\mu\left(t_{j}\right) C,
\end{array}
$$

and the vectors

$$
\begin{aligned}
\vec{F}^{(m)}\left(t_{j}\right)= & \left(F_{1}^{(m)}\left(t_{j}\right), \ldots, F_{N}^{(m)}\left(t_{j}\right)\right)^{T}, \\
\bar{b}_{j+1}^{(m)}= & \left(\bar{A}_{j}-\Delta t B_{j}^{(m)}-\frac{1}{2}(\Delta t)^{2} g_{0} C\right) \vec{u}_{j}^{(m)} \\
& -\frac{1}{2}(\Delta t)^{2} g_{1} C \vec{u}_{0}^{(m)}-(\Delta t)^{2} \sum_{\nu=1}^{j-1} g_{j-\nu} C \vec{u}_{\nu}^{(m)}+\Delta t \vec{F}^{(m)}\left(t_{j}\right) .
\end{aligned}
$$

(ii) Finding the vector $\vec{u}_{j+1}^{(m)}=\left(u_{1}^{(m)}\left(t_{j+1}\right), \ldots\right.$, $\left.u_{N}^{(m)}\left(t_{j+1}\right)\right)^{T}$ by solving the following system

$$
\bar{A}_{j} \vec{u}_{j+1}^{(m)}=\bar{b}_{j+1}^{(m)}
$$

When the process of computation is reached to $j=M-1$, we get

$$
\vec{u}_{j}^{(m)}=\left(u^{(m)}\left(x_{1}, t_{j}\right), \ldots, u^{(m)}\left(x_{N}, t_{j}\right)\right)^{T}, \quad 1 \leq j \leq M .
$$

(C4) The error of two consecutive steps of the iteration, at the $m^{\text {th }}$ step and at the $(m-1)^{\text {th }}$ step, is defined as follows:

$$
\left\|u^{(m)}-u^{(m-1)}\right\|_{M, N}=\max _{1 \leq j \leq M} \max _{1 \leq i \leq N}\left|u^{(m)}\left(x_{i}, t_{j}\right)-u^{(m-1)}\left(x_{i}, t_{j}\right)\right| .
$$

The process of the iteration will be stopped at the $m^{\text {th }}$ step when the following estimate is satisfied:

$$
\left\|u^{(m)}-u^{(m-1)}\right\|_{M, N}<10^{-4} .
$$

(C5) The error of the approximate solution (at the $m^{\text {th }}$ step) and the exact solution is defined by

$$
E_{M, N}=\left\|u^{(m)}-u_{e x}\right\|_{M, N}=\max _{1 \leq j \leq M} \max _{1 \leq i \leq N}\left|u^{(m)}\left(x_{i}, t_{j}\right)-u_{e x}\left(x_{i}, t_{j}\right)\right|,
$$


where $u_{e x}(x, t)$ is the exact solution. Next, we present an illustrated example and the corresponding numerical results in order to show that the convergence rate of the 2order iterative scheme is faster than that of the singleiterative scheme (which is schemes (32)-(34) with $\mathscr{F}^{(m)}(x, t)=f\left(x, t, u^{(m-1)}(x, t)\right)$, as in Remark 1).

For example, we consider problems (1)-(3) with

$$
\left\{\begin{array}{l}
f(x, t, u)=\rho|u|^{p-2} u+G(x, t), \\
\mu(t)=\mu_{*}+e^{-\bar{\mu} t}, \quad \mu_{*}>0, \bar{\mu}>0, \\
\alpha(t)=\alpha_{*}+e^{-\bar{\alpha} t}, \quad \alpha_{*}>0, \bar{\alpha}>0, \\
g(t)=g_{\max } e^{-\bar{g} t}, \quad g_{\max }>0,0<\bar{g} \neq 1, \\
G(x, t)=\left[1+\rho\left|x^{2}-\frac{5}{2} x+1\right|^{p-2} e^{-(p-2) t}\right]\left(x^{2}-\frac{5}{2} x+1\right) e^{-t} \\
-\left(4-\frac{5}{2 x}\right)\left[\alpha_{*}-\mu_{*}+e^{-\bar{\alpha} t}-e^{-\bar{\mu} t}+\frac{g_{\max }}{\bar{g}-1}\left(1-e^{-(\bar{g}-1) t}\right)\right] e^{-t}, \\
\widetilde{u}_{0}(x)=-x^{2}+\frac{5}{2} x-1 .
\end{array}\right.
$$

Then, $u_{e x}(x, t)=\left(-x^{2}+(5 / 2) x-1\right) e^{-t}$ is the exact solution of problems (1)-(3) corresponding to the constants $\zeta=1, R=2, p=4, \rho=10^{-2}, \alpha_{*}=\mu_{*}=\bar{\alpha}=\bar{\mu}=1$, and $\bar{g}=2$ and the given functions $f(x, t, u), \mu(t), \alpha(t)$, and $g(t)$ as in (88).

In case $\mathscr{F}^{(m)}(x, t)=f x, t, u^{(m-1)}(x, t)$, it means that $b^{(m)}(x, t)=D_{3} f\left(x, t, u^{(m-1)}(x, t)\right)=0$, and then the approximate scheme (8) has the form

$$
\left\{\begin{array}{l}
u_{0} \equiv 0, \\
\dot{u}^{(m)}(t)-\mu(t) L u^{(m)}-\alpha(t) L \dot{u}^{(m)}(t)+\int_{0}^{t} g(t-s) L u^{(m)}(s) \mathrm{d} s \\
=F^{(m)}(x, t), \quad 1<x<R, 0<t<T, \\
u_{x}^{(m)}(1, t)-h_{1} u^{(m)}(1, t)=u^{(m)}(R, t)=0, \\
u^{(m)}(x, 0)=\widetilde{u}_{0}(x),
\end{array}\right.
$$

where

$$
F^{(m)}(x, t)=f\left(x, t, u^{(m-1)}(x, t)\right) .
$$

Then, the matrix

$$
B_{j}^{(m)}=B^{(m)}\left(t_{j}\right)=-\mu\left(t_{j}\right) C \equiv B\left(t_{j}\right) \equiv B_{j}
$$

is independent of $m$. In this case, scheme (71) leads to the following approximate scheme, which is also called a singleiterative scheme

$$
\begin{aligned}
\bar{A}_{0} \vec{u}_{1}^{(m)} & =\left(\bar{A}_{0}-\Delta t B_{0}\right) \vec{u}_{0}^{(m)}+\Delta t \vec{F}^{(m)}\left(t_{0}\right) \equiv \bar{b}_{1}^{(m)}, \\
\bar{A}_{1} \vec{u}_{2}^{(m)} & =\left(\bar{A}_{1}-\Delta t B_{1}-\frac{1}{2}(\Delta t)^{2} g_{0} C\right) \vec{u}_{1}^{(m)}-\frac{1}{2}(\Delta t)^{2} g_{1} C \vec{u}_{0}^{(m)}+\Delta t \vec{F}^{(m)}\left(t_{1}\right) \equiv \bar{b}_{2}^{(m)}, \\
\bar{A}_{j} \vec{u}_{j+1}^{(m)} & =\left(\bar{A}_{j}-\Delta t B_{j}-\frac{1}{2}(\Delta t)^{2} g_{0} C\right) \vec{u}_{j}^{(m)}-\frac{1}{2}(\Delta t)^{2} g_{1} C \vec{u}_{0}^{(m)}-(\Delta t)^{2} \sum_{\nu=1}^{j-1} g_{j-\nu} C \vec{u}_{\nu}^{(m)}+\Delta t \vec{F}^{(m)}\left(t_{1}\right) \\
& \equiv \bar{b}_{j+1}^{(m)}, \quad 2 \leq j \leq M-1,
\end{aligned}
$$

where $\vec{F}^{(m)}\left(t_{j}\right)$ is determined by

$$
\left\{\begin{array}{l}
\vec{F}^{(m)}\left(t_{j}\right)=\left(F_{1}^{(m)}\left(t_{j}\right), \ldots, F_{N}^{(m)}\left(t_{j}\right)\right)^{T}, \\
F_{i}^{(m)}\left(t_{j}\right)=F^{(m)}\left(x_{i}, t_{j}\right)=f\left(x_{i}, t_{j}, u_{i}^{(m-1)}\left(t_{j}\right)\right) .
\end{array}\right.
$$

With the datum as in (88) and the error as in (86), the corresponding numerical results given by the single-iterative (92) and (93) and the 2-order iterative scheme are presented in Tables 1-5 and Figures 1-3.

According to the numerical results in Table 1, we can see that two iterative schemes used here are effective. Indeed,

(i) The third column of Table 1 shows that the errors of the approximate solution and the exact solution given by the single-iterative schemes (92) and (93) are decreased when $N$ and $M$ are increased.

(ii) The fourth column of Table 1 shows that the errors of the approximate solution and the exact solution given by the 2 -order scheme (71) are also decreased when $N$ and $M$ are increased.

(iii) The errors in the fourth column are less than these of the third column with the same grid $(N, M)$, respectively.

To compare the convergent speed of the single-iterative scheme and of 2-order iterative scheme, we establish the errors as in Tables 2-5. For more details, it is as follows.

First, with $M$ and $N$ fixed, we put the following errors. 
TABLE 1: Errors of the approximate solution and the exact solution.

\begin{tabular}{lccc}
\hline & & Single-iterative scheme & 2-order iterative scheme \\
\hline$N$ & $M$ & $E_{M, N}=\left\|u^{(m)}-u_{e x}\right\|_{M, N}$ & $E_{M, N}=\left\|u^{(m)}-u_{e x}\right\|_{M, N}$ \\
10 & 20 & 0.003574901202808 & 0.003574896182046 \\
20 & 40 & 0.001675675266970 & 0.001675670442791 \\
30 & 60 & 0.001092604754670 & 0.001092599898798 \\
40 & 80 & $8.098476439145630 \times 10^{-4}$ & $8.098428422048665 \times 10^{-4}$ \\
50 & 100 & $6.434079705471274 \times 10^{-4}$ & $6.434032161508618 \times 10^{-4}$ \\
\hline
\end{tabular}

TABLE 2: Errors of the approximate solution (at the $m^{\text {th }}$ step) and the exact solution, with $N=10$ and $M=20$.

\begin{tabular}{lcc}
\hline Number of iterations & Single-iterative scheme & 2-order iterative scheme \\
\hline$m$ & $E_{M, N}^{(m)}=\left\|u^{(m)}-u_{e x}\right\|_{M, N}$ & $E_{M, N}^{(m)}=\left\|u^{(m)}-u_{e x}\right\|_{M, N}$ \\
1 & 0.003609335483468 & 0.003609335483468 \\
2 & 0.003574901202808 & 0.003574896182046 \\
3 & 0.003574896182122 & 0.003574896181683 \\
4 & 0.003574896181684 & 0.003574896181683 \\
5 & 0.003574896181684 & 0.003574896181683 \\
6 & 0.003574896181684 & 0.003574896181683 \\
7 & 0.003574896181684 & 0.003574896181683 \\
8 & 0.003574896181684 & 0.003574896181683 \\
9 & 0.003574896181684 & 0.003574896181683 \\
10 & 0.003574896181684 & 0.003574896181683 \\
\hline
\end{tabular}

TABLE 3: Errors of two consecutive steps in each iteration, with $N=10$ and $M=20$.

\begin{tabular}{lcr}
\hline Number of iterations & Single-iterative scheme & 2-order iterative scheme \\
\hline$m$ & $D_{M, N}^{(m)}=\left\|u^{(m)}-u^{(m-1)}\right\|_{M, N}$ & $D_{M, N}^{(m)}=\left\|u^{(m)}-u^{(m-1)}\right\|_{M, N}$ \\
1 & 0.561983471074380 & 0.561983471074380 \\
2 & $3.700981639570555 \times 10^{-5}$ & $3.701583173776335 \times 10^{-5}$ \\
3 & $6.797891999310579 \times 10^{-9}$ & $6.646627692674656 \times 10^{-13}$ \\
4 & $8.078537838684952 \times 10^{-13}$ & $3.330669073875470 \times 10^{-16}$ \\
5 & $7.771561172376096 \times 10^{-16}$ & 0.0000000000000000 \\
6 & 0.0000000000000000 & 0.0000000000000000 \\
\hline
\end{tabular}

TABle 4: Errors of the approximate solution (at the $m^{\text {th }}$ step) and the exact solution, with $N=50$ and $M=100$.

\begin{tabular}{lrr}
\hline Number of iterations & Single-iterative scheme & 2-order iterative scheme \\
\hline$m$ & $E_{M, N}^{(m)}=\left\|u^{(m)}-u_{e x}\right\|_{M, N}$ & $E_{M, N}^{(m)}=\left\|u^{(m)}-u_{e x}\right\|_{M, N}$ \\
1 & $6.765921811121567 \times 10^{-4}$ & $6.765921811121567 \times 10^{-4}$ \\
2 & $6.434079705471274 \times 10^{-4}$ & $6.434032161508618 \times 10^{-4}$ \\
3 & $6.434032163748493 \times 10^{-4}$ & $6.434032158795233 \times 10^{-4}$ \\
4 & $6.434032159262637 \times 10^{-4}$ & $6.434032158795233 \times 10^{-4}$ \\
5 & $6.434032159262637 \times 10^{-4}$ & $6.434032158795233 \times 10^{-4}$ \\
6 & $6.434032159262637 \times 10^{-4}$ & $6.434032158795233 \times 10^{-4}$ \\
7 & $6.434032159262637 \times 10^{-4}$ & $6.434032158795233 \times 10^{-4}$ \\
8 & $6.434032159262637 \times 10^{-4}$ & $6.434032158795233 \times 10^{-4}$ \\
9 & $6.434032159262637 \times 10^{-4}$ & $6.434032158795233 \times 10^{-4}$ \\
10 & $6.434032159262637 \times 10^{-4}$ & $6.434032158795233 \times 10^{-4}$ \\
\hline
\end{tabular}

TABLE 5: Errors of two consecutive steps in each iteration, with $N=50$ and $M=100$.

\begin{tabular}{lcr}
\hline Number of iterations & Single-iterative scheme & 2-order iterative scheme \\
\hline$m$ & $D_{M, N}^{(m)}=\left\|u^{(m)}-u^{(m-1)}\right\|_{M, N}$ & $D_{M, N}^{(m)}=\left\|u^{(m)}-u^{(m-1)}\right\|_{M, N}$ \\
1 & 0.562475970780469 & 0.562475970780469 \\
2 & $3.613601710050762 \times 10^{-5}$ & $3.614203006274685 \times 10^{-5}$ \\
3 & $6.811192498901164 \times 10^{-9}$ & $6.195877144676842 \times 10^{-13}$ \\
4 & $8.621436897726653 \times 10^{-13}$ & $1.337818744673314 \times 10^{-14}$ \\
5 & $1.412758798835512 \times 10^{-14}$ & 0.0000000000000000 \\
6 & 0.0000000000000000 & 0.0000000000000000 \\
\hline
\end{tabular}




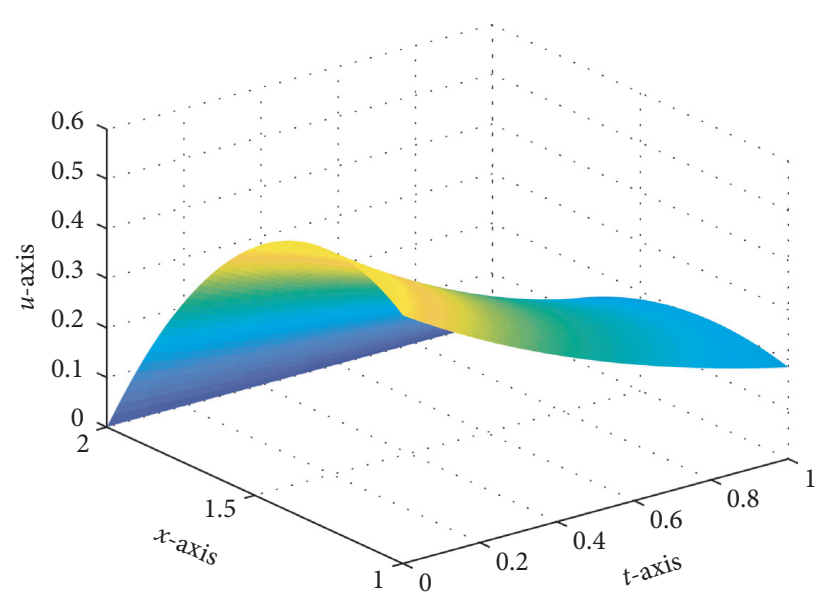

FIgURE 1: The surface of the finite-difference approximate solution of $u^{(m)}(x, t)$ defined by the 2-order iterative scheme (8), with respect to equation (71) and the grid of $N=50$ and $M=100$ as mentioned above.

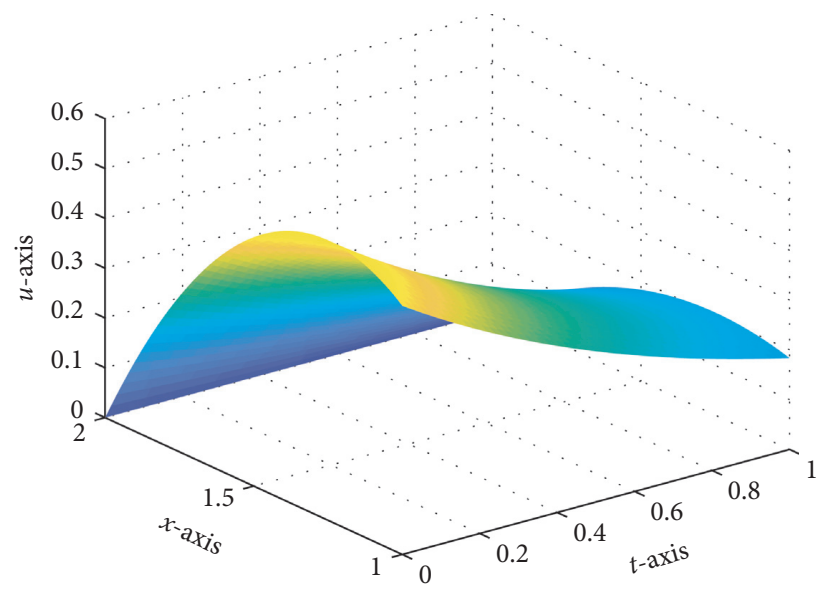

FIgURE 2: The surface of the finite-difference approximate solution of $u^{(m)}(x, t)$ defined by the single-iterative scheme (89), with respect to equation (92) and the grid of $N=50$ and $M=100$ as mentioned above.

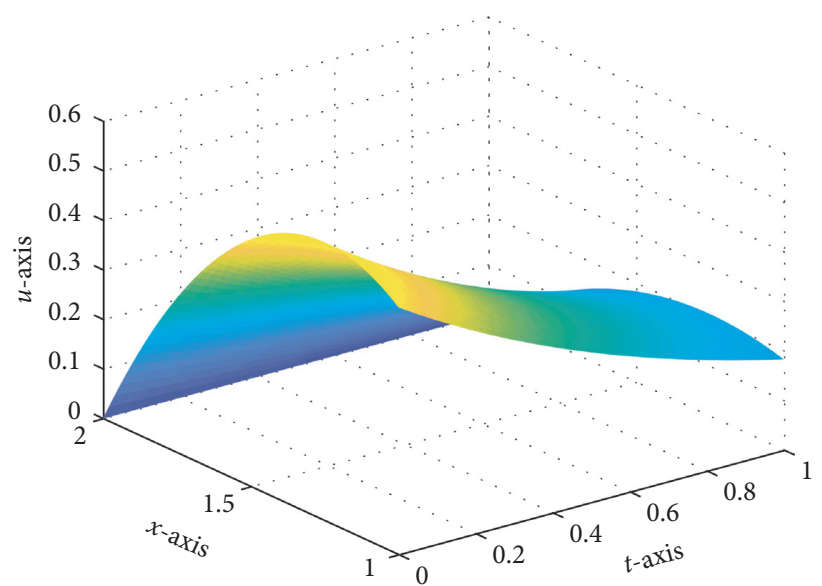

FIgURE 3: The exact solution of problems (1)-(3) given by $u_{e x}(x, t)=\left(-x^{2}+(5 / 2) x-1\right) e^{-t}$, with the input datum as in (88). 
(i) The error of the approximate solution (at the $m^{\text {th }}$ step) and the exact solution:

$$
E_{M, N}^{(m)}=\left\|u^{(m)}-u_{e x}\right\|_{M, N}=\max _{1 \leq j \leq M} \max _{1 \leq i \leq N}\left|u^{(m)}\left(x_{i}, t_{j}\right)-u_{e x}\left(x_{i}, t_{j}\right)\right| .
$$

(ii) The error of two consecutive steps of the iteration, at the $m^{\text {th }}$ step and at the $(m-1)^{\text {th }}$ step:

$$
\begin{aligned}
D_{M, N}^{(m)}= & \left\|u^{(m)}-u^{(m-1)}\right\|_{M, N}=\max _{1 \leq j \leq M} \max _{1 \leq i \leq N} \mid u^{(m)}\left(x_{i}, t_{j}\right) \\
& -u^{(m-1)}\left(x_{i}, t_{j}\right) \mid .
\end{aligned}
$$

Next, we compute the errors as in Tables $2-5$. The errors $E_{M, N}^{(m)}$ of two iterative schemes according to the iterative steps are given in Tables 2 and 4, where the grids are considered with $N=10, M=20$ and $N=50, M=100$ respectively. Similarly, the errors $D_{M, N}^{(m)}$ of two iterative schemes are given in Tables 3 and 5.

According to the numerical results in Tables 2-5, we have the following:

(i) The values of the error $E_{M, N}^{(m)}$ given in the columns 2 and 3 of Table 4 are decreased when the iterative steps are increased from 1 to 10 . It is reasonable by the fact that both schemes are convergent.

(ii) The values of the third column are less than these of the second column, line by line, in Tables 2 and 4 . This shows that the convergent speed of 2-order iterative scheme is faster than that of the single-iterative scheme. It is similar to the errors $D_{M, N}^{(m)}$ of two schemes given in Tables 3 and 5 .

Finally, we have drawn the approximated solutions and the exact solution of problems (1)-(3) with the datum as in (88).

\section{Conclusion}

This paper has proved the solvability of problems (1)-(3) for a nonlinear pseudoparabolic align with Robin-Dirichlet conditions by establishing an approximate sequence $\left\{u^{(m)}\right\}$ based on a high-order iterative scheme or a single-iterative scheme. The proposed schemes are tested on an example in which a standard finite-difference scheme is used suitably. The numerical results obtained here show that the convergence rate of the 2-order iterative scheme is faster than that of the single-iterative scheme. Because of the efficient convergence rate, the present high-order iterative scheme offers a good alternative to find a solution of nonlinear problems for partial differential aligns.

\section{Data Availability}

Research data used in this study are available from the references.

\section{Conflicts of Interest}

The authors declare that they have no conflicts of interest.

\section{Authors' Contributions}

All authors contributed equally to this article. They read and approved the final manuscript.

\section{Acknowledgments}

This research was funded by NTTU Foundation for Science and Technology Development.

\section{References}

[1] R. E. Showalter and T. W. Ting, "Pseudoparabolic partial differential equations," SIAM Journal on Mathematical Analysis, vol. 1, no. 1, pp. 1-26, 1970.

[2] R. E. Showalter and T. W. Ting, "Asymptotic behavior of solutions of pseudoparabolic partial differential aligns," Annali di Matematica Pura ed Applicata, vol. 90, no. 4, pp. 241-258, 1971.

[3] R. E. Showalter, "Existence and representation theorems for a semilinear Sobolev equation in Banach space," SIAM Journal on Mathematical Analysis, vol. 3, no. 3, pp. 527-543, 1972.

[4] R. E. Showalter, "Hilbert space methods for partial differential aligns," Electronic Journal of Differential Equations Monograph 01, 1994.

[5] C. J. Amick, J. L. Bona, and M. E. Schonbek, "Decay of solutions of some nonlinear wave equations," Journal of Differential Equations, vol. 81, no. 1, pp. 1-49, 1989.

[6] S. Asghar, T. Hayat, and P. D. Ariel, "Unsteady Couette flows in a second grade fluid with variable material properties," Communications in Nonlinear Science and Numerical Simulation, vol. 14, no. 1, pp. 154-159, 2009.

[7] G. Barenblat, I. Zheltov, and I. Kochiva, "Basic concepts in the theory of seepage of homogeneous liquids in fissured rocks," Journal of Applied Mathematics and Mechanics, vol. 24, no. 5, pp. 1286-1303, 1960.

[8] T. B. Benjamin, J. L. Bona, and J. J. Mahony, "Models align of long waves in nonlinear dispersive systems," Philosophical Transactions of the Royal Society A, vol. 272, no. 1220, pp. 47-78, 1972.

[9] T. Hayat, M. Khan, and M. Ayub, "Some analytical solutions for second grade fluid flows for cylindrical geometries," Mathematical and Computer Modelling, vol. 43, no. 1-2, pp. 16-29, 2006.

[10] T. Hayat, F. Shahzad, and M. Ayub, "Analytical solution for the steady flow of the third grade fluid in a porous half space," Applied Mathematical Modelling, vol. 31, no. 11, pp. 24242432, 2007.

[11] T. W. Ting, "Certain non-steady flows of second-order fluids," Archive for Rational Mechanics and Analysis, vol. 14, no. 1, pp. 1-26, 1963.

[12] M. Renardy, W. J. Hrusa, and J. A. Nohel, Mathematical Problems in Viscoelasticity, Vol. 35, Longman Higher Education, London, UK, 1987.

[13] A. Bouziani, "Solvability of nonlinear pseudoparabolic align with a nonlocal boundary condition," Nonlinear Anal, vol. 55, no. 7-8, pp. 883-904, 2003.

[14] A. Bouziani, "Initial-boundary value problems for a class of pseudoparabolic equations with integral boundary 
conditions," Journal of Mathematical Analysis and Applications, vol. 291, no. 2, pp. 371-386, 2004.

[15] D.-Q. Dai and Y. Huang, "A moment problem for one-dimensional nonlinear pseudoparabolic equation," Journal of Mathematical Analysis and Applications, vol. 328, no. 2, pp. 1057-1067, 2007.

[16] E. Shivanian and M. Aslefallah, "Stability and convergence of spectral radial point interpolation method locally applied on two-dimensional pseudoparabolic equation," Numerical Methods for Partial Differential Equations, vol. 33, no. 3, pp. 724-741, 2017.

[17] F. Sun, L. Liu, and Y. Wu, "Global existence and finite time blow-up of solutions for the semilinear pseudo-parabolic equation with a memory term," Applicable Analysis, vol. 98, no. 4, pp. 735-755, 2019.

[18] L. X. Truong, L. T. P. Ngoc, A. P. N. Dinh, and N. T. Long, "Existence, blow-up and exponential decay estimates for a nonlinear wave equation with boundary conditions of twopoint type," Nonlinear Analysis: Theory, Methods \& Applications, vol. 74, no. 18, pp. 6933-6949, 2011.

[19] X. Zhu, F. Li, and Y. Li, "Global solutions and blow up solutions to a class of pseudo-parabolic equations with nonlocal term," Applied Mathematics and Computation, vol. 329, pp. 38-51, 2018.

[20] L. T. P. Ngoc, L. X. Truong, and N. T. Long, "High-order iterative methods for a nonlinear Kirchhoff wave align," Demonstratio Mathematica, vol. 43, no. 3, pp. 605-634, 2010.

[21] L. T. P. Ngoc, L. H. K. Son, and N. T. Long, "A N-order iterative scheme for a nonlinear Carrier wave in an annular with Robin-Dirichlet conditions," Nonlinear Functional Analysis and Applications, vol. 22, no. 1, pp. 147-169, 2017.

[22] L. X. Truong, L. T. P. Ngoc, and N. T. Long, "High-order iterative schemes for a nonlinear Kirchhoff-Carrier wave align associated with the mixed homogeneous conditions," Nonlinear Analysis: Theory, Methods \& Applications, vol. 71, no. 12, pp. 467-484, 2009.

[23] L. X. Truong, L. T. P. Ngoc, and N. T. Long, "The n-order iterative schemes for a nonlinear Kirchhoff-Carrier wave equation associated with the mixed inhomogeneous conditions," Applied Mathematics and Computation, vol. 215, no. 5, pp. 1908-1925, 2009.

[24] G. F. Pinder, Numerical Methods for Solving Partial Differential Aligns, Wiley, Hoboken, NJ, USA, 2018.

[25] A. V. Gulin and A. Y. Mokin, "Stability of a family of weighted finite-difference schemes," Computational Mathematics and Modeling, vol. 20, no. 2, pp. 152-172, 2009.

[26] J. Jachimavičienè and M. Sapagovas, "Locally one-dimensional difference scheme for a pseudoparabolic align with nonlocal conditions," Lithuanian Mathematical Journal, vol. 52, no. 1, pp. 53-61, 2012.

[27] J. Jachimavičienè, Ž. Jesevičiūte, and M. Sapagovas, "The stability of finite-difference schemes for a pseudoparabolic align with nonlocal conditions," Numerical Functional Analysis and Optimization, vol. 35, no. 10, pp. 988-1001, 2014.

[28] L. T. P. Ngoc, N. A. Triet, A. P. Ngoc Dinh, and N. T. Long, "Existence and exponential decay of solutions for a wave equation with integral nonlocal boundary conditions of memory type," Numerical Functional Analysis and Optimization, vol. 38, no. 9, pp. 1173-1207, 2017.

[29] A. M. Oberman, "Convergence rate for difference schemes for polyhedral nonlinear parabolic equations," Journal of Computational Mathematics, vol. 28, no. 4, pp. 474-488, 2010.

[30] N. T. Long, A. P. N. Dinh, and L. X. Truong, "Existence and decay of solutions of a nonlinear viscoelastic problem with a mixed nonhomogeneous condition," Numerical Functional Analysis and Optimization, vol. 29, no. 11-12, pp. 1363-1393, 2008.

[31] I. Amirali, G. M. Amiraliyev, M. Cakir, and E. Cimen, "Explicit finite difference methods for the delay pseudoparabolic aligns," The Scientific World Journal, vol. 2014, Article ID 497393, 7 pages, 2014.

[32] M. K. Beshtokov, "Finite-difference method for a nonlocal boundary value problem for a third-order pseudoparabolic equation," Differential Equations, vol. 49, no. 9, pp. 1134-1141, 2013.

[33] M. K. Beshtokov, "On the numerical solution of a nonlocal boundary value problem for a degenerating pseudoparabolic equation," Differential Equations, vol. 52, no. 10, pp. 1341-1354, 2016.

[34] M. Brachet and J.-P. Chehab, "Stabilized times schemes for high accurate finite differences solutions of nonlinear parabolic equations," Journal of Scientific Computing, vol. 69, no. 3, pp. 946-982, 2016.

[35] J. E. Macías-Díaz, I. E. Medina-Ramírez, and A. Puri, "Numerical treatment of the spherically symmetric solutions of a generalized Fisher-Kolmogorov-Petrovsky-Piscounov equation," Journal of Computational and Applied Mathematics, vol. 231, no. 2, pp. 851-868, 2009.

[36] J. E. Macías-Díaz, "On a boundedness-preserving semi-linear discretization of a two-dimensional nonlinear diffusion-reaction model," International Journal of Computer Mathematics, vol. 89, no. 12, pp. 1678-1688, 2012.

[37] J. E. Macías-Díaz and A. Szafrańska, "Existence and uniqueness of monotone and bounded solutions for a finitedifference discretization à la Mickens of the generalized Burgers-Huxley equation," Journal of Difference Equations and Applications, vol. 20, no. 7, pp. 989-1004, 2014.

[38] J. E. Macías-Díaz and A. E. González, "A convergent and dynamically consistent finite-difference method to approximate the positive and bounded solutions of the classical Burgers-Fisher equation," Journal of Computational and Applied Mathematics, vol. 318, pp. 604-615, 2017.

[39] R. Jiwari, "A Haar wavelet quasilinearization approach for numerical simulation of Burgers' equation," Computer Physics Communications, vol. 183, no. 11, pp. 2413-2423, 2012.

[40] R. Jiwari, "A hybrid numerical scheme for the numerical solution of the Burgers' equation," Computer Physics Communications, vol. 188, pp. 59-67, 2015.

[41] R. Jiwari, S. Pandit, and M. E. Koksal, "A class of numerical algorithms based on cubic trigonometric B-spline functions for numerical simulation of nonlinear parabolic problems," Computational \& Applied Mathematics, vol. 38, p. 140, 2019.

[42] A. Szafrańska and J. E. Macías-Díaz, "On the convergence of a finite-difference discretization a la Mickens of the generalized Burgers-Huxley align," Journal of Difference Equations and Applications, vol. 20, no. 10, pp. 1444-1451, 2014.

[43] O. P. Yadav and R. Jiwari, "Finite element analysis and approximation of Burgers'-Fisher equation," Numerical Methods for Partial Differential Equations, vol. 33, no. 5, pp. 1652-1677, 2017.

[44] J. L. Lions, Quelques Méthodes de Résolution des Problèmes Aux Limites Non-Linéaires, Dunod: Gauthier-Villars, Paris, France, 1969.

[45] L. Zhang, "Decay of solution of generalized Benjamin-BonaMahony-Burgers equations in n-space dimensions," Nonlinear Analysis: Theory, Methods \& Applications, vol. 25, no. 12, pp. 1343-1369, 1995. 